\title{
Vincristine and bortezomib use distinct upstream mechanisms to activate a common SARM1-dependent axon degeneration program
}

\author{
Stefanie Geisler, ${ }^{1,2}$ Ryan A. Doan, ${ }^{1}$ Galen C. Cheng, ${ }^{1}$ Aysel Cetinkaya-Fisgin, ${ }^{3}$ Shay X. Huang, ${ }^{1}$ \\ Ahmet Höke, ${ }^{3}$ Jeffrey Milbrandt, ${ }^{2,4}$ and Aaron DiAntonio ${ }^{2,5}$ \\ 'Department of Neurology, Washington University School of Medicine in St. Louis, St. Louis, Missouri, USA. ${ }^{2}$ Hope Center \\ for Neurological Disorders, Washington University, St. Louis, Missouri, USA. ${ }^{3}$ Department of Neurology, Johns Hopkins \\ University, Baltimore, Maryland, USA. ${ }^{4}$ Department of Genetics and ${ }^{5}$ Department of Developmental Biology, Washington \\ University School of Medicine in St. Louis, St. Louis, Missouri, USA.
}

Chemotherapy-induced peripheral neuropathy is one of the most prevalent dose-limiting toxicities of anticancer therapy. Development of effective therapies to prevent chemotherapy-induced neuropathies could be enabled by a mechanistic understanding of axonal breakdown following exposure to neuropathy-causing agents. Here, we reveal the molecular mechanisms underlying axon degeneration induced by 2 widely used chemotherapeutic agents with distinct mechanisms of action: vincristine and bortezomib. We showed previously that genetic deletion of SARM1 blocks vincristine-induced neuropathy and demonstrate here that it also prevents axon destruction following administration of bortezomib in vitro and in vivo. Using cultured neurons, we found that vincristine and bortezomib converge on a core axon degeneration program consisting of nicotinamide mononucleotide NMNAT2, SARM1, and loss of NAD+ but engage different upstream mechanisms that closely resemble Wallerian degeneration after vincristine and apoptosis after bortezomib. We could inhibit the final common axon destruction pathway by preserving axonal NAD+ levels or expressing a candidate gene therapeutic that inhibits SARM1 in vitro. We suggest that these approaches may lead to therapies for vincristine- and bortezomib-induced neuropathies and possibly other forms of peripheral neuropathy.

Conflict of interest: $S C, J M, A D$, and Washington University are inventors on patents related to this work. AD and $J M$ are cofounders of Disarm Therapeutics, and AD, JM, and AH are shareholders in Disarm Therapeutics and are compensated for serving on its scientific advisory board.

Copyright: (c) 2019 American Society for Clinical Investigation

Submitted: April 30, 2019

Accepted: August 1, 2019

Published: September 5, 2019

Reference information: /CI Insight. 2019;4(17):e129920. https://doi. org/10.1172/jici.insight.129920.

\section{Introduction}

Chemotherapy-induced peripheral neuropathy (CIPN) is one of the most common complications of cancer treatment, affecting up to $80 \%$ of cancer patients (1). CIPN is characterized by burning pain, numbness, tingling, imbalance, impaired dexterity, and weakness in the hands and feet. In contrast with other side effects, CIPN can extend well beyond the duration of treatment and cause permanent disability ( 2 , 3). Despite major efforts, therapies to prevent or effectively treat CIPN remain elusive $(4,5)$. Most forms of CIPN are caused by axonal degeneration, but the mechanisms of axonal breakdown remain unknown for many neuropathy-causing agents $(6,7)$. In addition, it is unknown whether distinct agents engage different death programs or converge on a shared axon degeneration pathway to induce symptoms (6). This lack of a clear mechanistic understanding of the disorder is a major impediment to the development of efficacious therapies to prevent CIPN.

In contrast with axon degeneration in CIPNs, exciting breakthroughs have been made in recent years unraveling molecular mechanisms of axon degeneration after traumatic injury $(8,9)$, which gives hope that those insights can be applied to understanding CIPN (10). Wallerian degeneration, as axon degeneration after traumatic injury is called, refers to rapid axonal fragmentation after a long period of relative latency due to a genetically encoded self-destruction program that is activated distal to the nerve cut. Sterile alpha and toll/interleukin-1 receptor motif-containing 1 (SARM1) is a central determinant of this program because activation of SARM1 triggers irreversible commitment to Wallerian degeneration $(11,12)$. SARM1 is a multidomain protein that consists of an autoinhibitory $\mathrm{N}$ terminus, tandem 
SAM domains that mediate constitutive homomultimerization, and an executioner TIR domain (12). Upon injury, N-terminal inhibition is relieved, potentially via a rise in nicotinamide mononucleotide (NMN) secondary to NMN adenylyltransferase 2 (NMNAT2) loss (13-15), which thereby activates the intrinsic SARM1 TIR NADase $(12,16)$. Active SARM1 cleaves the metabolic cofactor nicotinamide adenine dinucleotide (NAD+), generating nicotinamide and the calcium-mobilizing agents ADPR and cADPR (16), leading to local $\mathrm{NAD}^{+}$depletion, followed by metabolic collapse and axon fragmentation (17). Activation of SARM1 is sufficient to induce axon degeneration, even in healthy axons, whereas genetic deletion of SARM1 protects axons from degeneration for more than 2 weeks after nerve cut in vivo, indicating that SARM1 is necessary and sufficient to induce axon degeneration after axotomy.

After nerve transection all axons distal to the cut degenerate nearly simultaneously, whereas in chronic neuropathies only a few short axonal segments degenerate at any given time in a pattern that proceeds slowly from distal to proximal. Thus, axon loss during chemotherapy may be due to different injury responses than after axotomy. Encouragingly, we discovered that SARM1-KO is also protective in models of peripheral neuropathy induced by the chemotherapeutic agents vincristine and paclitaxel $(18,19)$. Vincristine and paclitaxel inhibit intraaxonal transport (20) and thus produce axon degeneration conceptually similar to the complete cessation of axonal transport after nerve cut. It is not known whether SARM1 deletion blocks axon degeneration caused by chemotherapeutic agents with different mechanisms of action or whether the same upstream regulators and downstream effectors of SARM1 are engaged in neuropathy as after axotomy. To address these questions, we investigated the molecular mechanisms of axon degeneration precipitated by 2 commonly used chemotherapeutic agents, vincristine and bortezomib (BTZ). Vincristine inhibits tubulin polymerization and affects axonal transport, whereas BTZ inhibits the proteasome $(7,21)$. Neuropathy is the major nonhematological side effect of both, affecting up to $80 \%$ of patients treated with vincristine (22) and $30 \%-65 \%$ of patients receiving BTZ (23).

We demonstrate here that genetic deletion of SARM1 strongly decreases not only vincristine-induced axon degeneration but also axonal destruction following administration of BTZ in vitro and in vivo. Vincristine and BTZ administration engage distinct upstream pathways that converge on a core axonal degeneration program consisting of NMNAT2, SARM1, and NAD ${ }^{+}$. We are able to inhibit this program, and thus pathological axon degeneration, by expression of a candidate gene therapeutic targeting SARM1 and suggest that such a therapeutic may be of value in the prevention of vincristine- and BTZ-induced CIPN and possibly other forms of peripheral neuropathies.

\section{Results}

Vincristine and BTZ cause axon degeneration by distinct mechanisms. To study molecular mechanisms underlying axon degeneration after vincristine and BTZ administration, cultured dorsal root ganglion (DRG) neurons were treated after long neurites were established on day in vitro (DIV) 7 with $40 \mathrm{nM}$ vincristine or 100 nM BTZ. Axon fragmentation of proximal and distal axons was quantified over time using high-throughput imaging and an automated axon degeneration index (24). After vincristine application, axons began to degenerate at 12 hours (Figure 1A) and were completely fragmented by 36 hours (Figure 1, A and B). In contrast, axon degeneration began later after BTZ administration, between 24 and 36 hours (Figure 1, $\mathrm{A}$ and $\mathrm{B}$ ). Once initiated, the process proceeded rapidly, and axons were completely fragmented within 48 hours after BTZ addition (Figure 1, A and B).

To characterize axon degeneration caused by vincristine and BTZ, we cultured DRG neurons in compartmentalized microfluidic chambers that allow independent manipulation of cell bodies and axons (25, 26). When vincristine was added to the axon compartment, axon fragmentation was complete by 48 hours (Figure 1, C and E). However, axons remained intact for days when vincristine was applied exclusively to the cell body compartment (Figure 1, C and E, and ref. 26) In contrast, when BTZ was administered to axons, they remained intact for more than 48 hours (Figure 1, D and E), whereas they degenerated within 48 hours when BTZ was added to the cell bodies (Figure 1, D and E). Taken together, these data indicate that vincristine stimulates an axon autonomous degenerative process, whereas BTZ causes axon degeneration only after exposure of the cell body.

SARM1 is required for both vincristine- and BTZ-induced axon degeneration. The loss of SARM1 potently inhibits vincristine-induced axon degeneration in cultured neurons and in vivo $(18,27)$. To test whether genetic deletion of SARM1 similarly prevents BTZ-induced axon degeneration, WT and SARM1KO DRG neurons were treated with either vincristine or BTZ and axon degeneration was measured. 
A

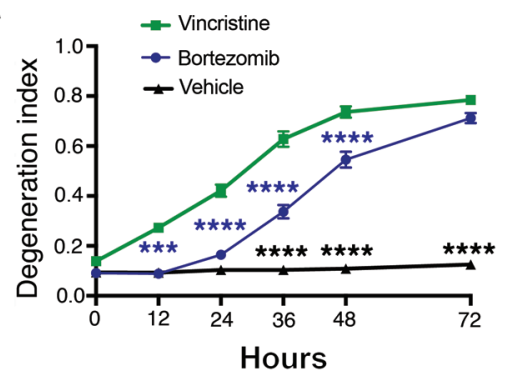

C

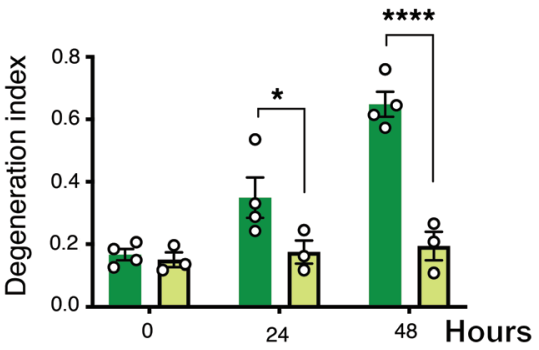

Vincristine added to
B

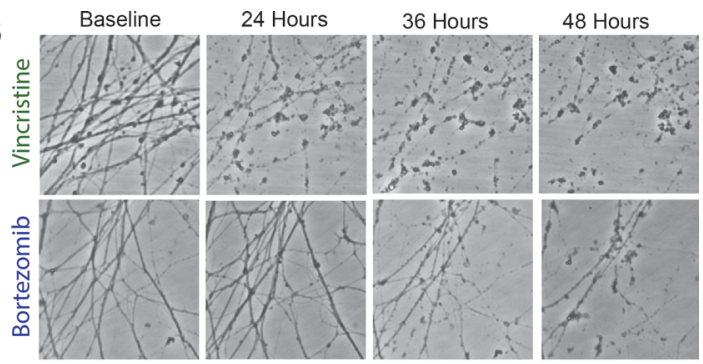

D

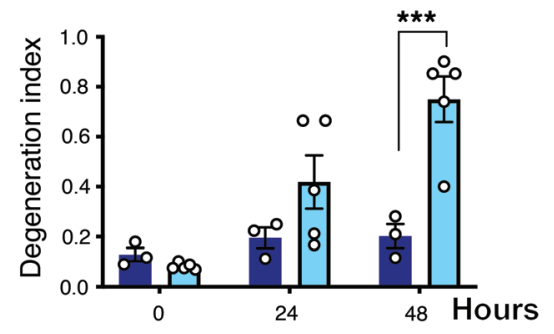

E

$\square$ Soma chamber
Bortezomib added to Axon chamber Soma chamber

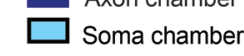

Vincristine
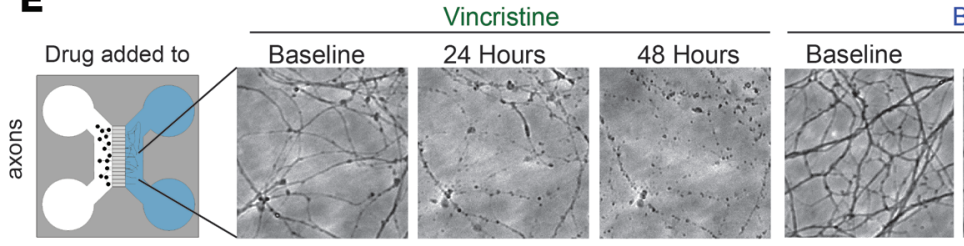

Bortezomib
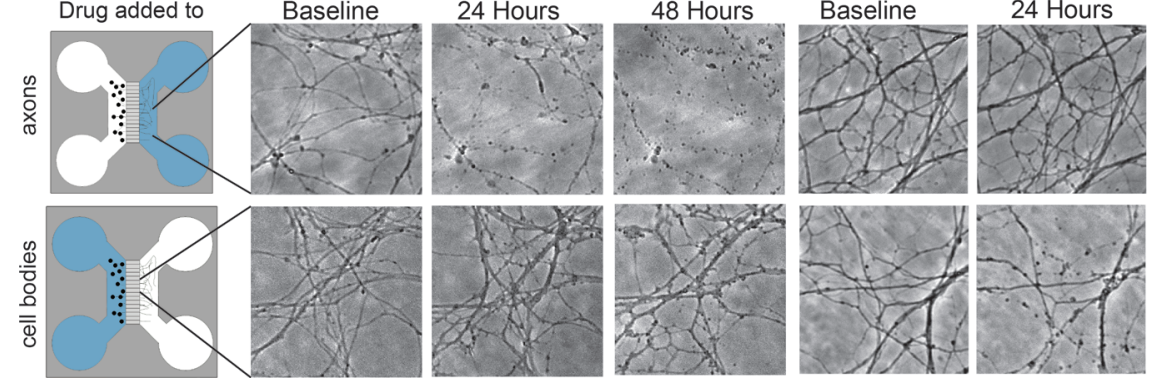

48 Hours

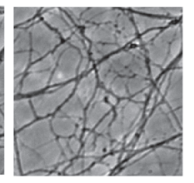

Figure 1. Vincristine and BTZ induce axon degeneration by distinct mechanisms. (A) DRG neurons were treated with $40 \mathrm{nM}$ vincristine or $100 \mathrm{nM}$ BTZ, and axons imaged using high-throughput automated imaging at indicated time points. Axon degeneration was quantified using a degeneration index, which ranges from 0 (perfectly intact) to 1 (perfectly fragmented). Data were tested with a 2-way ANOVA showing significant main effects of group $F$ $(2,185)=484.8, P<0.0001$; time $F(5,185)=213.4, P<0.0001$; and interaction $F(10,185)=58.28$; post hoc Šídák's multiple-comparisons test, ${ }^{* * *} P=$ 0.0001 vincristine versus BTZ 12 hours (blue asterisks); ${ }^{* * *} P<0.0001$ vincristine versus $B T Z$ (blue asterisks), vincristine versus vehicle, and $B T Z$ versus vehicle (black asterisks); $P=0.3102 \mathrm{BTZ}$ versus vehicle at 24 hours $(n=13)$. (B) Representative phase-contrast photomicrographs of axons treated with vincristine or BTZ taken at indicated time points showing axon degeneration 24 hours after vincristine but not BTZ administration. Instead, axons fragmented between 36 and 48 hours after BTZ. Original magnification 200x. (C) DRG neurons were grown in compartmentalized microfluidic chambers, and $40 \mathrm{nM}$ vincristine was added to the compartment containing cell bodies (soma chamber, $n=3$ experiments) or axons (axon chamber, $n=4$ experiments) and degeneration in the axon compartment determined at indicated times. A 2-way ANOVA showed significant main effects of group $F(1,5)=34.49$, $P=0.002$; time $F(2,10)=18.96, P=0.0004$; and interaction $F(2,10)=13.29, P=0.0015$. Šídák's multiple-comparisons test, ${ }^{*} P<0.05,{ }^{* * * *} P<0.0001$. (D) DRG neurons were grown in compartmentalized chambers, and $100 \mathrm{nM} \mathrm{BTZ} \mathrm{was} \mathrm{added} \mathrm{to} \mathrm{the} \mathrm{soma} \mathrm{(} n=5$ experiments) or axon compartment ( $n=$ 3 experiments) and degeneration in the axon compartment determined over time. Two-way ANOVA showed significant main effects of group $F(1,6)=$ 8.389, $P=0.0275$; time $F(2,12)=16.39, P=0.0004$; and interaction $F(2,12)=10.55, P=0.0023$. Šídák's multiple-comparisons test, ${ }^{* * *} P<0.001$. (E) Representative bright-field photomicrographs of the axon compartment taken at indicated times after vincristine or BTZ was added to axon compartment or cell body chamber. Original magnification 200x.

Axons of SARM1-KO DRG neurons remained intact for at least 72 hours after vincristine administration (Figure 2A). Similarly, axons of SARM1-KO neurons were morphologically intact for at least 72 hours after BTZ administration, whereas WT axons were completely fragmented by 48 hours (Figure 2B). We recently developed a SARM1 dominant-negative (SARM1-DN) mutant as a candidate gene therapeutic for disorders of axon loss (27). Expression of the SARM1-DN mutant potently inhibits WT SARM1 function after nerve transection and in a cellular model of vincristine-induced neuropathy (27). To test whether this SARM1 genetic inhibitor protects from BTZ-induced axon degeneration, we used lentivirus to express the 
SARM1-DN mutant in WT DRG neurons. Akin to SARM1-KO axons, axons of WT neurons expressing the SARM1-DN mutant were protected from BTZ-induced degeneration for at least 72 hours (Figure 2, C and D), suggesting that inhibition of SARM1 may be a viable strategy to prevent BTZ-induced axon loss.

Such a strategy is useful only if the axons also remain metabolically active. We thus used the cell-permeable red fluorescent dye TMRM to probe for metabolic activity. TMRM is sequestered by healthy mitochondria and loses its fluorescent signal upon loss of the mitochondrial potential. As expected, TMRM fluorescence was no longer observed in axons of WT DRG neurons 72 hours after BTZ application (Figure 2D). In contrast, TMRM staining was detected well beyond 72 hours in axons of SARM1-KO neurons and in WT neurons expressing the SARM1-DN mutant (Figure 2D), indicating that the SARM1-deficient axons remained metabolically active as well as morphologically intact.

We next examined whether SARM1 deletion is also protective in an in vivo model of BTZ-induced neuropathy. WT and SARM1-KO mice on the same genetic background were injected intravenously with $0.8 \mathrm{mg} / \mathrm{kg}$ BTZ or vehicle twice weekly for 4 weeks. In human patients, BTZ causes predominantly a sensory peripheral neuropathy characterized by a decrease of unmyelinated or thinly myelinated axons in the epidermis (23). In accord with the human data, we observed a significant decrease of intraepidermal nerve fiber (IENF) density in BTZ-treated mice (Figure 2, E and F). In contrast, the IENF density in SARM1-KO mice was similar between vehicle- and BTZ-treated mice (Figure 2, E and F), indicating that SARM1-KO prevents the degeneration of intraepidermal axons after BTZ. These data together with published reports $(12,16,18)$ indicate that SARM1 is necessary for both vincristine- and BTZ-induced axon degeneration in vitro and in vivo.

Vincristine and BTZ induce SARM1-dependent depletion of axonal $N A D^{+}$. Activation of SARM1 after nerve cut causes a rapid, dramatic decrease of $\mathrm{NAD}^{+}$, which is followed by local metabolic collapse and axon degeneration (17). To investigate whether $\mathrm{NAD}^{+}$decreases in the cell body or axon after vincristine or BTZ treatment, we extracted and measured $\mathrm{NAD}^{+}$from axon or cell body lysates. We observed a dramatic drop of axonal $\mathrm{NAD}^{+}$concentration between 4 and 12 hours after vincristine and BTZ treatment, which was before axon fragmentation became apparent (Figure 3, A and B). In contrast with axonal $\mathrm{NAD}^{+}$levels, $\mathrm{NAD}^{+}$levels in the soma decreased slowly and to a lesser extent after vincristine and BTZ treatment (Figure 3, A and B). In SARM1-KO DRG neurons, the rapid $\mathrm{NAD}^{+}$drop in the axon was abolished following vincristine and BTZ administration (Figure 3, A and B). Instead, NAD ${ }^{+}$in SARM1-KO axons declined gradually and with similar kinetics as in the somata of WT and SARM1-KO DRGs. These results indicate that exposure to these chemotherapeutic agents results in SARM1 activation in the axon, which causes a rapid decrease of $\mathrm{NAD}^{+}$before axon fragmentation is initiated.

SARM1-induced axon degeneration after axotomy can be blocked by maintaining $\mathrm{NAD}^{+}$levels in the distal axon $(9,28)$. We therefore investigated whether maintaining $\mathrm{NAD}^{+}$is also sufficient to protect from vincristine- and BTZ-induced axon degeneration. One of the most potent manipulations to increase $\mathrm{NAD}^{+}$ levels in DRG neurons is to express nicotinamide riboside kinase 1 (NRK1) in the presence of nicotinamide riboside (NR; ref. 28). NRK1 is an enzyme in the $\mathrm{NAD}^{+}$salvage pathway (Figure 3C) that phosphorylates NR to NMN (29), which is then converted to $\mathrm{NAD}^{+}$by NMNAT. Indeed, lentivirus-mediated expression of NRK1 in DRG neurons and preincubation with NR increased NAD ${ }^{+}$levels by about 10-fold in our hands (control: $0.15 \pm 0.05 \mu \mathrm{M} \mathrm{NAD}^{+} ; n=34$ wells, 8 independent experiments; NRK1+NR: $1.7 \pm 0.4$ $\mu \mathrm{M} \mathrm{NAD}^{+}, n=15$ wells, 3 independent experiments; unpaired $t$ test; $\left.P<0.0001\right)$. Whereas $\mathrm{NAD}^{+}$was not detectable in axon lysates 24 hours after vincristine and BTZ (Figure 3, A and B), in the presence of overexpressed NRK1 and NR, axonal NAD ${ }^{+}$levels remained high even 48 hours after vincristine $(0.7 \pm 0.2 \mu \mathrm{M}$ $\mathrm{NAD} ; n=10$ wells, 2 independent experiments $)$ and BTZ administration $\left(0.9 \pm 0.2 \mu \mathrm{M} \mathrm{NAD}^{+} ; n=9\right.$ wells, 2 independent experiments) (Figure 3D). This increase of $\mathrm{NAD}^{+}$was associated with robust protection of axons from vincristine- and BTZ-induced degeneration (Figure 3, E-H), which lasted at least 72 hours after drug administration. As seen after axotomy (28), less robust protection from vincristine and BTZ was observed after lentivirus-mediated overexpression of nicotinamide phosphoribosyltransferase (NAMPT) (Figure 3, E-H), another enzyme in the $\mathrm{NAD}^{+}$salvage pathway (Figure $3 \mathrm{C}$ ).

Vincristine and BTZ induce loss of axonal NMNAT2. In the intact axon, SARM1 is inhibited by the NAD ${ }^{+}$-synthesizing enzyme NMNAT2 (30). NMNAT2 has a short half-life and constant delivery from the cell body into the axon is necessary for axon survival (31). After axotomy, NMNAT2 levels drop quickly in the distal axon, promoting degeneration (31). To determine whether vincristine and BTZ administration also cause a loss of axonal NMNAT2, we examined axon-only lysates from DRG neurons using Western blot analysis. We found 
A

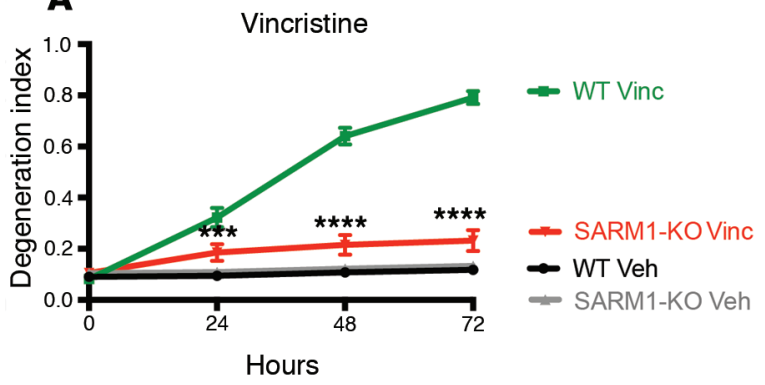

C

SARM1-DN

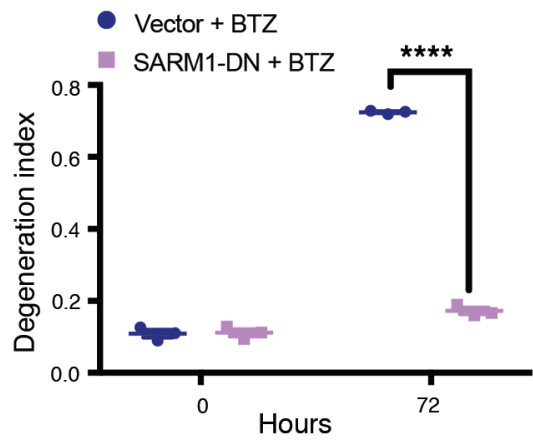

E

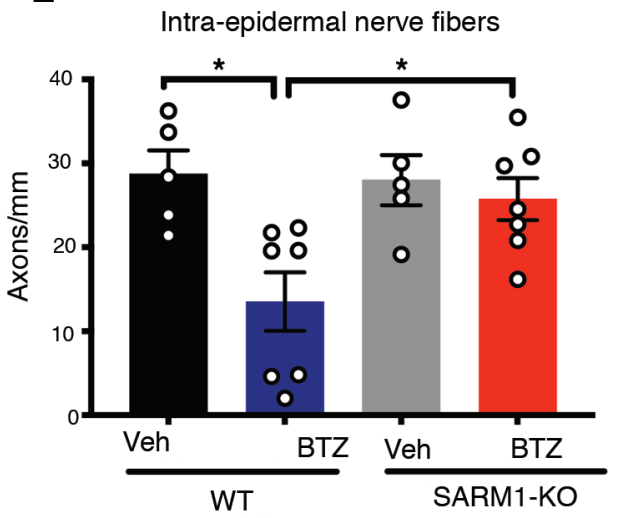

$\mathbf{F}$

\section{B}

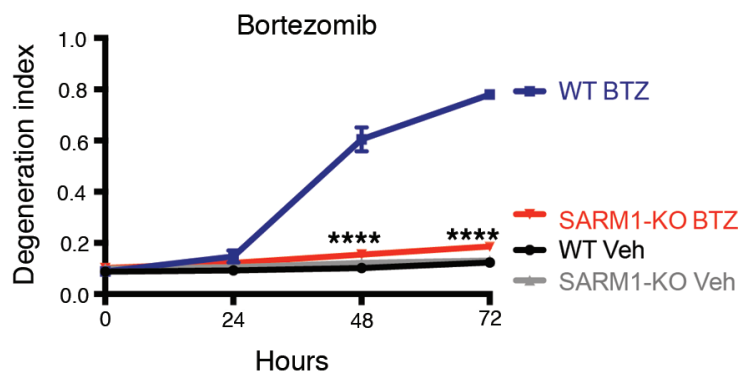

D

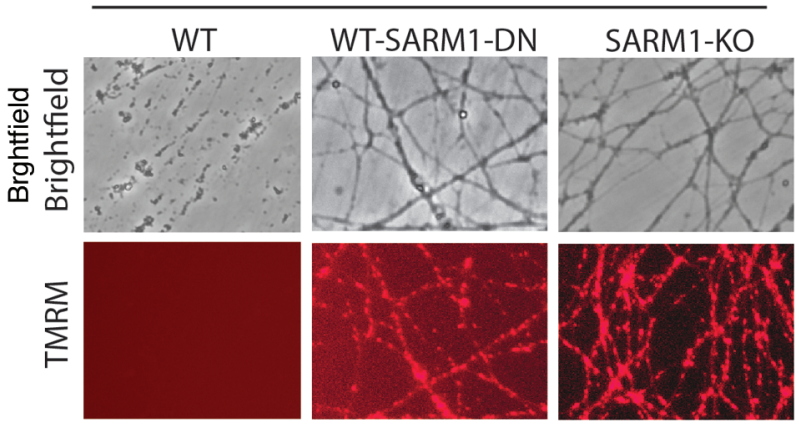

Vehicle

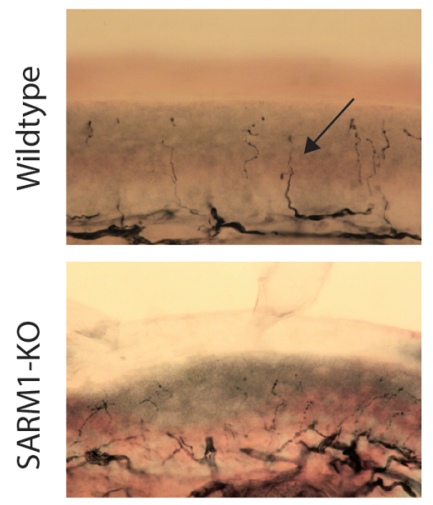

BTZ

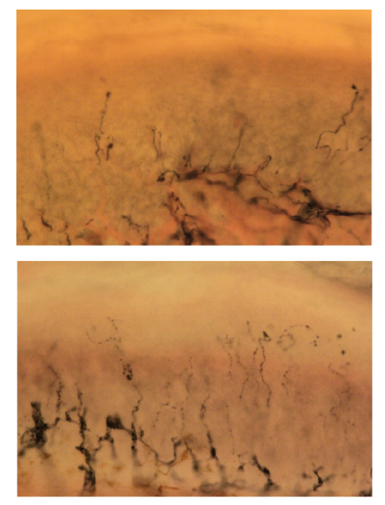

Figure 2. SARM1 is required for both vincristine- and BTZ-induced axon degeneration. (A) WT or SARM1-KO DRG neurons were treated with 40 nM vincristine or vehicle and axon degeneration determined over time. Degeneration index ranges from 0 (completely intact) to 1 (completely fragmented). A 2-way ANOVA showed significant main effects of group $F(3,16)=76.43, P<0.0001$; time $F(3,48)=198.8, P<0.0001$; and interaction $F(9$, $48)=110.2, P<0.0001$. Tukey's multiple-comparisons test, ${ }^{* *} P<0.001 \mathrm{WT}$ vincristine versus SARM1-K0 vincristine 24 hours, ${ }^{* * * *} P<0.0001$ ( $n$ = 5). (B) WT or SARM1-KO DRG neurons were treated with $100 \mathrm{nM} \mathrm{BTZ} \mathrm{or} \mathrm{vehicle} \mathrm{and} \mathrm{axon} \mathrm{degeneration} \mathrm{determined} \mathrm{at} \mathrm{indicated} \mathrm{times.} \mathrm{A} \mathrm{2-way}$ ANOVA showed significant main effects of group $F(3,16)=175.2, P<0.0001$; time $F(3,48)=290.8, P<0.0001$; and interaction $F(9,48)=180.2, P<$ 0.0001. Post hoc Tukey's multiple-comparisons test, ${ }^{* * * *} P<0.0001$ WT BTZ versus SARM1-KO BTZ $(n=5)$. (C) WT DRG neurons expressing a control vector (vector) or SARM1 dominant-negative (SARM1-DN) transgene were treated with $100 \mathrm{nM} \mathrm{BTZ}$ and axon degeneration was determined. A 2-way ANOVA showed main effects of group $F(1,4)=1301, P<0.001$; time $F(1,4)=1235, P<0.0001$; and interaction $F(1,4)=832.2, P<0.0001$; Šídák's multiple-comparisons test, ${ }^{* * *} P<0.0001(n=3)$. (D) Representative bright-field micrographs of WT axons, WT axons expressing the SARM1-DN transgene, and SARM1-KO axons taken 72 hours after BTZ was added. The mitochondrial potential was monitored with red tetramethylrhodamine (TMRM) fluorescence in the same axons as shown above. Original magnification 200x. (E) WT or SARM1-KO mice were treated for 4 weeks with intravenous BTZ ( $n=7$ per group) or vehicle ( $n=5$ per group), and the intraepidermal nerve fiber (IENF) density of the footpads was determined. There was significantly less IENF in BTZ-treated WT mice compared with vehicle-treated WT and BTZ-treated SARM1-KO mice. WT vehicle: 28.7 \pm 3.2 , and $n=5$; WT BTZ: $13.5 \pm 3.8$, and $n=7$; SARM1-KO vehicle: $28.0 \pm 3.4$, and $n=5$; SARM1-KO BTZ: $25.8 \pm 2.7$, and $n=7$; One-way ANOVA, $F(3,20)=$ 5.827 and $P=0.0050$; post hoc Tukey's, ${ }^{*} P<0.05$. (F) Representative images of IENF densities stained with the pan-neuronal marker PGP 9.5 (black, arrow) of WT (top row) or SARM1-KO (bottom row) mice treated with vehicle (left) or BTZ (right). Original magnification 400x. 

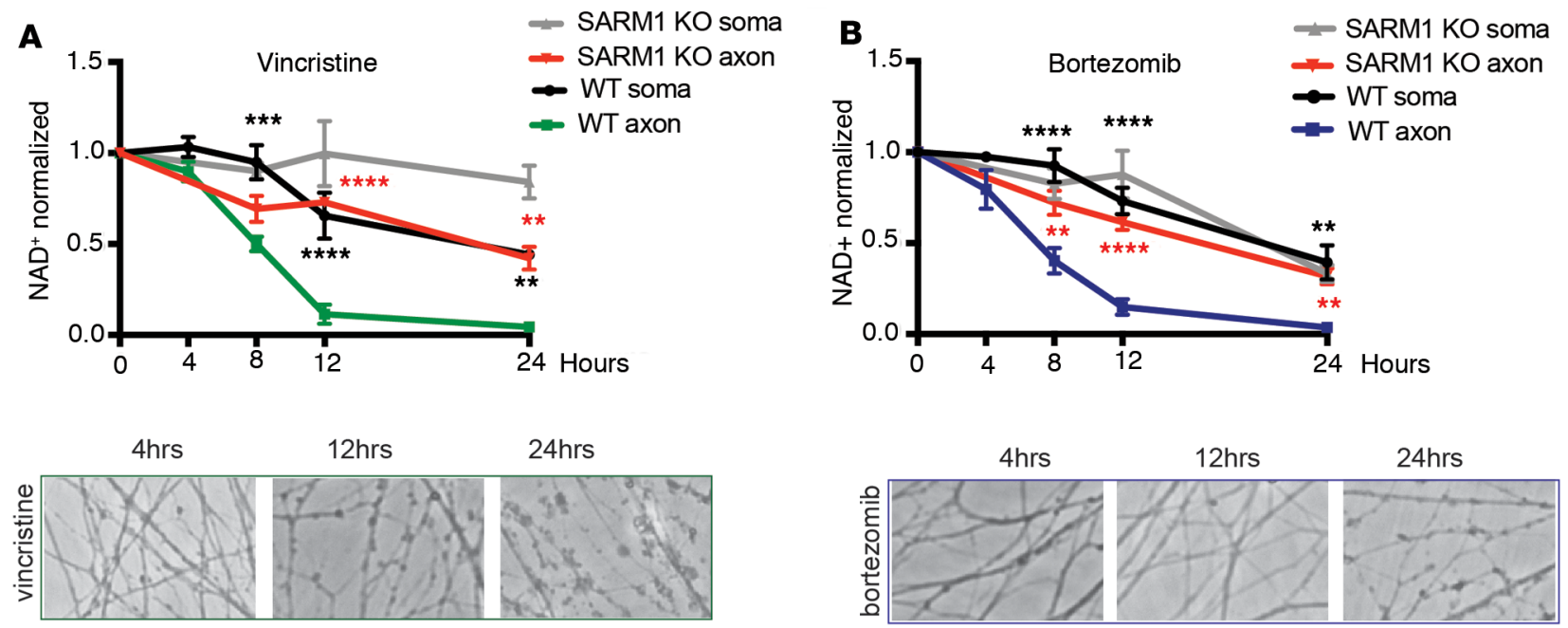

C

NAD+

(nicotinamide adenine dinucleotide)

NMNAT 1-3

NMN

(nicotinamide mononucleotide)

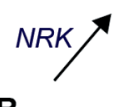

NR

(nicotinamide riboside)

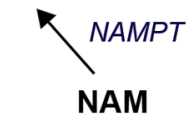

(nicotinamide)
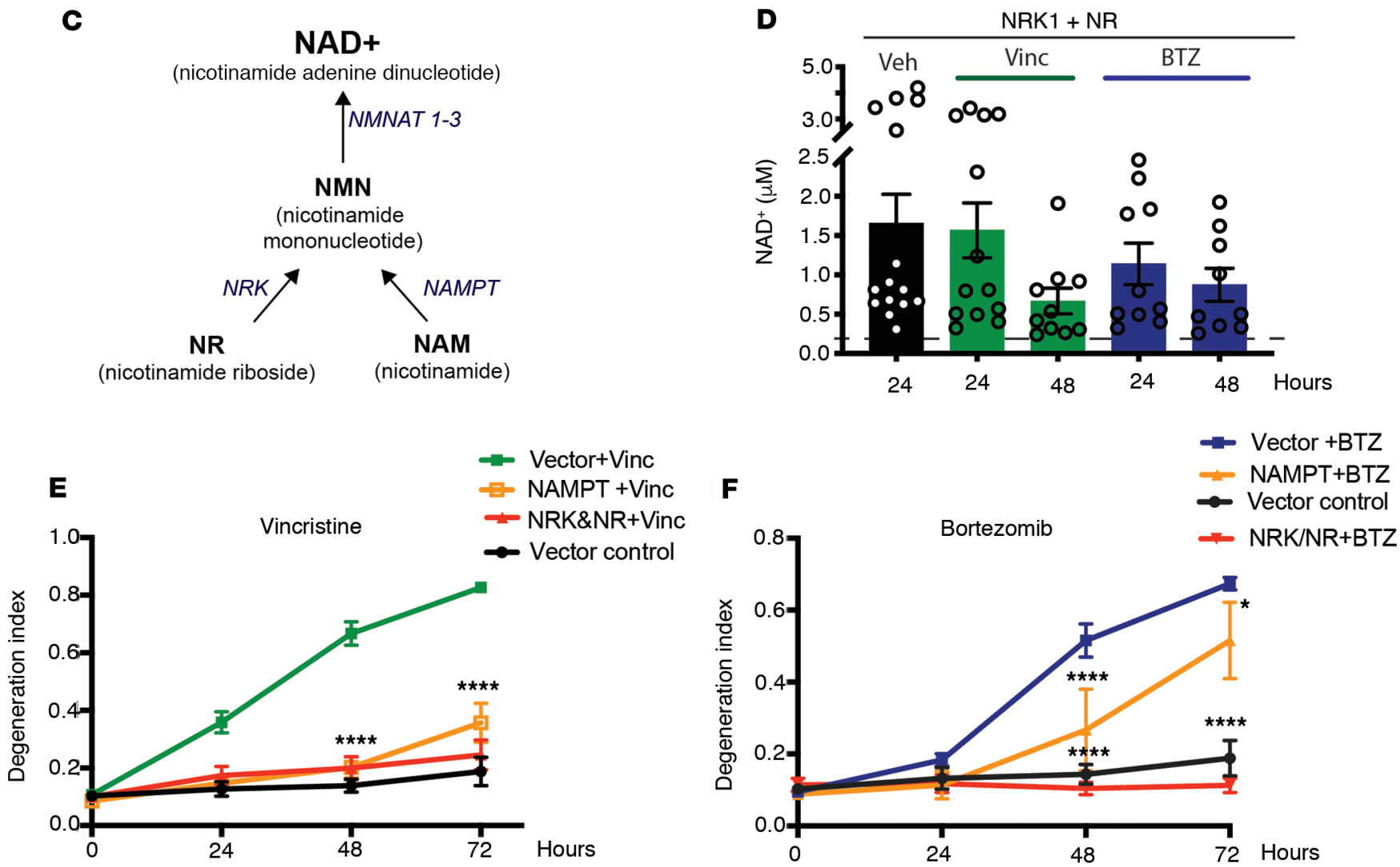

G Vincristine

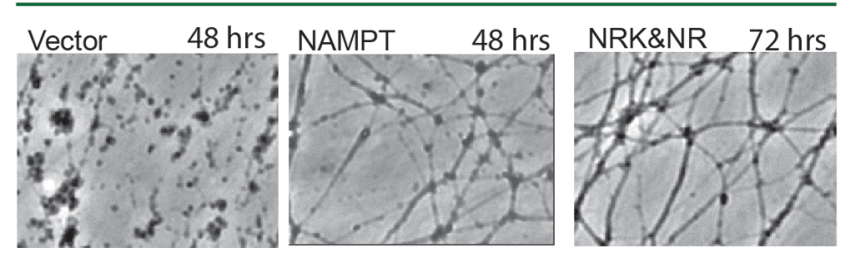

H Bortezomib

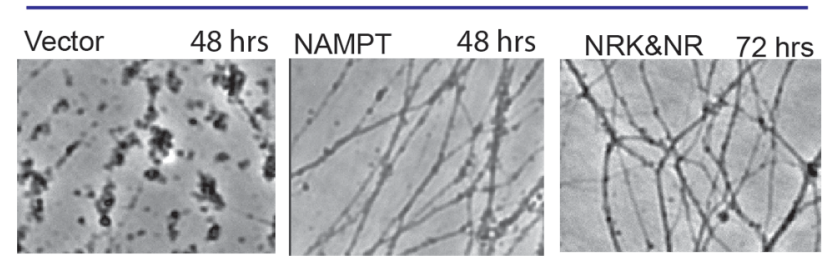

Figure 3. Vincristine and BTZ induce SARM1-dependent depletion of axonal NAD+. (A) HPLC was used to measure NAD+ levels in WT and SARM1-KO neurons from axon or cell body extracts and normalized to baseline. Two-way ANOVA shows main effect for group $F(3,8)=52, P<0.0001$; time $F(3,24)=39.77, P<$ 0.0001 ; and interaction $F(9,24)=5.797, P=0.0003$. Tukey's multiple-comparisons test, ${ }^{* *} P<0.01,{ }^{* * *} P<0.001,{ }^{* * *} P<0.0001$. Black asterisks, WT axon versus WT soma; red asterisks, WT axon versus SARM1-KO axon $(n=3)$. Representative bright-field images just before NAD+ extraction. Original magnification 200x. (B) NAD+ levels were measured as described in $\mathbf{A}$. Two-way ANOVA showed main effects of group $F(3,46)=30.23, P<0.0001$; time $F(3,46)=88$.83, $P<0.0001$; and interaction $F(9,46)=5, P=0.0001$. Tukey's multiple-comparisons test, ${ }^{* *} P<0.01,{ }^{* * *} P<0.0001 ; n=3-5$ per time point. Representative 
bright-field images just before NAD+ extraction. Original magnification 200x. (C) Diagram of selected mammalian NAD+ biosynthesis pathways. NMNAT, nicotinamide mononucleotide adenylyltransferase; NAMPT, nicotinamide phosphoribosyltrans ferase; NRK, nicotinamide riboside kinase. (D) WT DRG neurons expressing NRK1 were treated with $1 \mathrm{mM}$ NR and vincristine, BTZ, or vehicle and NAD+ was measured from axon-only lysates . Axonal NAD+ concentration of DRG

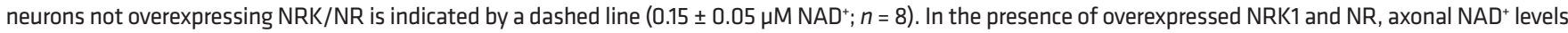
remained high 48 hours after vincristine administration. (E) WT DRG neurons expressing indicated constructs were treated with vincristine, and axon degeneration was determined. Two-way ANOVA showed main effects of group $F(3,15)=32.82, P<0.001$; time $F(3,45)=134.9, P<0.0001$; and interaction $F(9,45)=44, P$ $<0.0001$. Tukey's multiple-comparisons test, ${ }^{* * *} P<0.0001$ ( $n=5$ for NRK/NR, $n=3$ for NAMPT). (F) DRG neurons expressing indicated constructs were treated with BTZ. Two-way ANOVA showed main effects of group $F(3,17)=21.13, P<0.001$; time $F(3,51)=97.13, P<0.0001$; and interaction $F(9,51)=35.95, P<0.0001$. Tukey's multiple-comparisons test, ${ }^{* * *} P<0.0001$; ${ }^{*} P<0.05$ ( $n=5$ NRK/NR, $n=3$ NAMPT). (G and $\left.\mathbf{H}\right)$ Representative bright-field photomicrographs of axons expressing the indicated constructs after vin $\neg$ cristine $(\mathbf{G})$ or BTZ (H) administration. Original magnification 200×.

that axonal NMNAT2 levels decreased significantly between 8 and 12 hours after vincristine application and continued to decline over time (Figure 4A). Similarly, axonal NMNAT2 levels dropped within the first 8 hours after BTZ treatment and rebounded slightly around 24 hours, after irreversible commitment to axon degeneration had occurred (Figure 4B). Thus, axonal NMNAT2 decreases before axons start to fragment in response to vincristine or BTZ exposure. If a decrease of NMNAT2 activates SARM1 after BTZ and vincristine administration, continued expression of NMNAT should protect from vincristine- and BTZ-induced axon degeneration. Accordingly, lentiviral-mediated expression of cytosolic NMNAT1 (32), a stable NMNAT isoform that substitutes in axons for the short-lived NMNAT2, resulted in robust protection from both vincristine- and BTZ-induced axon degeneration (Figure 4, C and D), indicating that the rapid decrease of $\mathrm{NAD}^{+}$in the axon after vincristine and BTZ administration is mediated by SARM1 activation. These results indicate SARM1 acts via the same downstream effector (i.e., $\mathrm{NAD}^{+}$) and is subject to the same immediate upstream regulator (i.e., NMNAT2) in axons damaged mechanically or by diverse chemotherapeutic agents.

Vincristine- but not BTZ-induced axon degeneration is modulated by the MAPK pathway. The neuronal stress MAPK pathway mediated by dual leucine zipper kinase (DLK) and leucine zipper-bearing kinase (LZK) promotes Wallerian degeneration $(33,34)$ by speeding the turnover of $\operatorname{NMNAT} 2(35,36)$. To further explore whether the molecular pathways regulating BTZ- and vincristine-induced axon degeneration are distinct or identical, we investigated whether they are differentially affected by inhibiting this MAPK cascade. Consistent with previous reports that genetic deletion or pharmacological inhibition of DLK protects against axon degeneration after vincristine administration $(33,36)$, we found that $1 \mu \mathrm{M}$ of the DLK inhibitor GNE-3511 provided substantial protection against vincristine-induced axon degeneration (Figure 5A). To our surprise, however, GNE-3511 did not protect from BTZ-induced degeneration (Figure 5B). As an independent test of this unexpected result, we used lentivirus to express previously validated guide RNAs directed against the mitogen-activated protein double kinases MKK4 and MKK7 (35), which function downstream of DLK and LZK, in Cas9-knockin DRG neurons. Similar to inhibiting DLK, deletions of MKK4 and MKK7 resulted in axon protection after vincristine (Figure 5C) but not BTZ (Figure 5D) administration.

Inhibition of DLK suppresses axotomy-induced axon degeneration by increasing NMNAT2 levels (Figure $5 \mathrm{E}$ and ref. 35). After treatment with vincristine, but not BTZ, we observed an increase of axonal NMNAT2 levels when DLK was inhibited (Figure 5E), likely explaining the selective protection following treatment with vincristine but not after BTZ treatment. The mechanism by which the DLK pathway regulates NMNAT2 is not known, and so it is unclear how vincristine and BTZ differentially influence this regulatory mechanism. Regardless, these findings identify a mechanistic distinction between vincristine- and BTZ-induced axon degeneration.

Axon degeneration stimulated by bortezomib is transcriptionally regulated and mediated by axonal activated caspases. Our finding that DLK/LZK and MKK4/MKK7 promote vincristine but not BTZ-induced axon degeneration suggests that vincristine and BTZ engage different upstream pathways that converge on SARM1. Because BTZ-induced axon degeneration is mediated via effects on the soma, we hypothesized that BTZ-induced degeneration is transcriptionally regulated. To evaluate the role of transcription in vincristine- and BTZ-induced degeneration, we examined BTZ-treated DRG neurons cultured in the presence of the transcriptional inhibitor actinomycin $(1 \mu \mathrm{g} / \mathrm{mL})$. We found that BTZ-stimulated axon degeneration was decreased for 72 hours (Figure 6B). In contrast, actinomycin had no effect on axon degeneration induced by vincristine (Figure 6A).

A classic example of a transcriptionally regulated death program is caspase-dependent axon degeneration after nerve growth factor (NGF) deprivation (37). Underlying this axon death pathway is a caspase cascade beginning with Bax and resulting in the ordered activation of caspase-9, -3 , and -6 (38). If BTZ treatment leads to activation of a caspase-dependent axon death program, then inhibiting caspases should decrease BTZ-induced degeneration. Indeed, using a cell-permeable, irreversible pan-caspase inhibitor, Z-VAD(OMe)-FMK at 
A

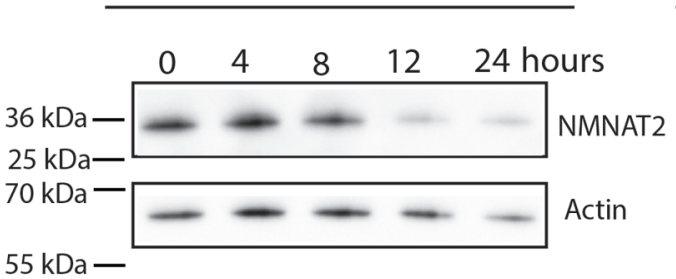

B Bortezomib

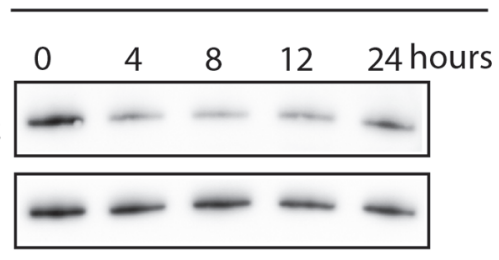

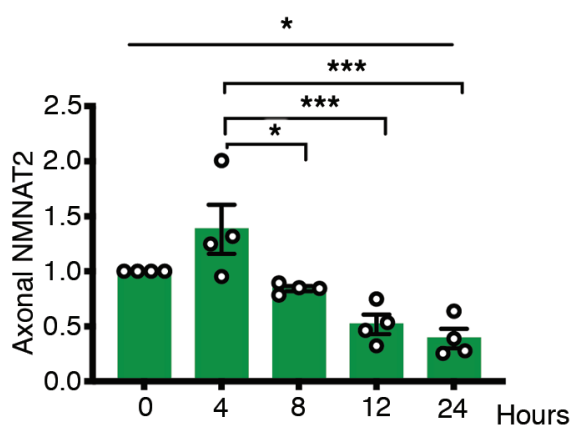
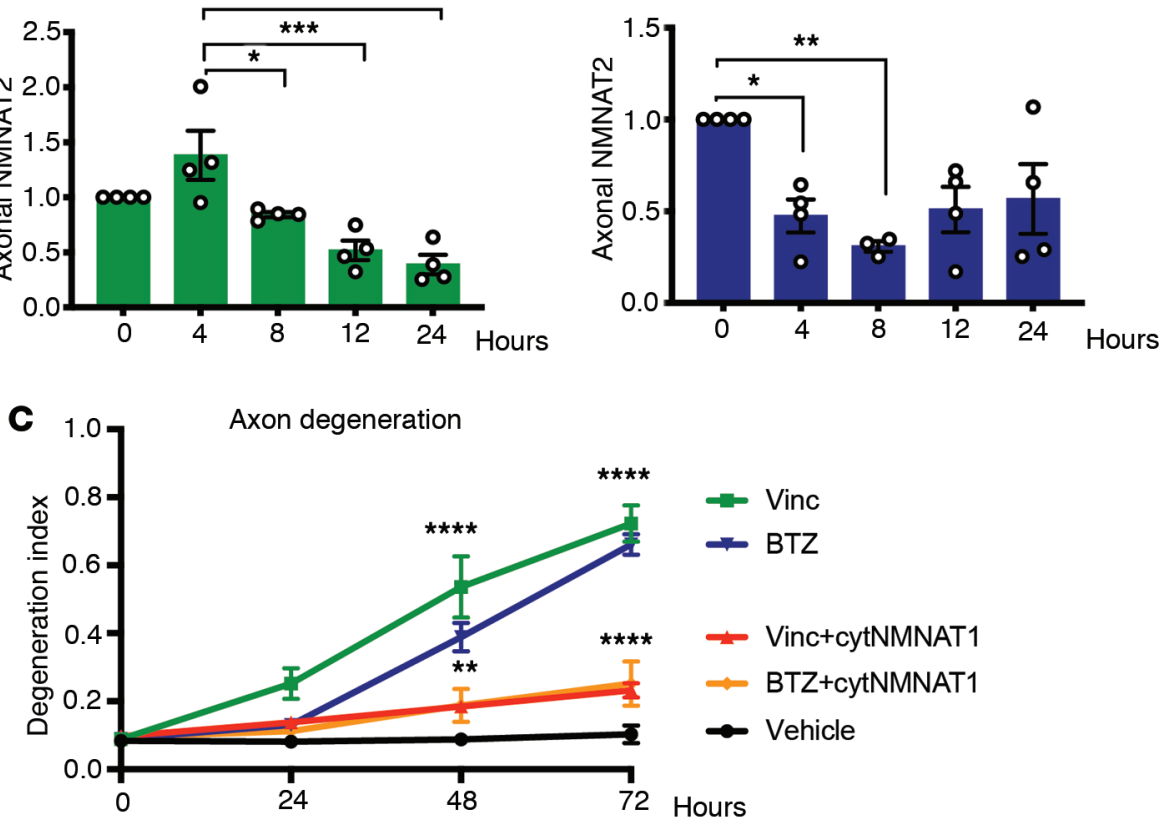

Figure 4. Axonal NMNAT2 levels decrease after vincristine and BTZ administration. (A) Axon-only extracts were derived from DRG neurons at indicated time points after adding vincristine and immunoblotted for endogenous NMNAT2 or actin (loading control). Quantification of endogenous NMNAT2 protein levels normalized to actin and baseline showed a decrease of axonal NMNAT2 over time. One-way ANOVA $F(4,15), P=0.0002$; post hoc Tukey's ${ }^{*} P<0.05,{ }^{* * *} P<0.001 ; n=$ 4. (B) Axon extracts derived from DRG neurons at indicated time points after adding BTZ were immunoblotted for endogenous NMNAT2 and actin. Quantification of NMNAT2 levels as in A showed a decrease of axonal NMNAT2 4 and 8 hours after BTZ. One-way ANOVA $F(4,14)=$ 4.839; $P=0.0116$. Post hoc Tukey's ${ }^{*} P<0.05$, ${ }^{*} P<0.01 ; n=4$. (C) DRG neurons expressing cytoplasmic NMNAT1 (cytNMNAT1) were treated with vincristine or BTZ, and axon degeneration was determined at indicated time points using the degeneration index. Expression of cytNMNAT1 prevented vincristine- and BTZ-induced axon degeneration. Two-way ANOVA showed significant main effects of group $F$ (4, $10)=21.44, P<0.0001$; time $F(3,30)=148.5$, $P<0.0001$; and interaction $F(12,30)=26.14$. Post-hoc analysis with Tukey's multiple-comparisons test, ${ }^{* *} P<0.01,{ }^{* * * *} P<0.0001(n=$ 3). (D) Representative phase-contrast microphotographs of axons expressing cytNMNAT1 showing intact axons 72 hours after adding vincristine or BTZ. Original magnification 200x.

\section{D}

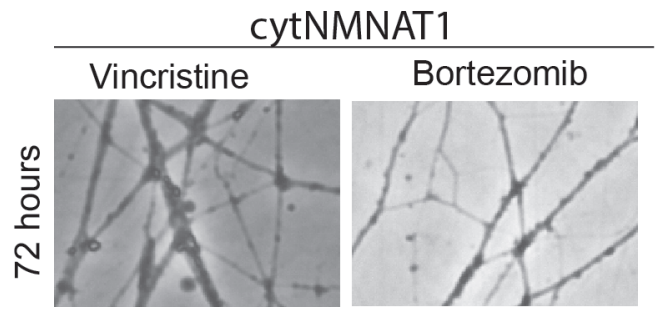

$100 \mu \mathrm{M}$, a concentration that effectively blocks NGF deprivation-induced axon degeneration of DRG neurons (data not shown), we found that axon degeneration in response to BTZ was inhibited (Figure 6D), but that axon degeneration stimulated by vincristine proceeded unabated (Figure 6C).

We next evaluated whether caspase-3, an essential executioner caspase in apoptosis, is activated in axons after BTZ. Unfortunately, selective caspase-3 inhibitors do not block NGF deprivation-induced axon degeneration $(39,40)$. We therefore used immunohistochemistry to detect caspase- 3 activation. We found cleaved caspase-3 immunoreactivity in axons of DRG neurons within 16 hours after BTZ administration, before initiation of axonal fragmentation (Figure $6 \mathrm{E}$ ). In contrast, we did not observe cleaved caspase- 3 immunoreactivity 16 hours after vincristine or vehicle administration (Figure 6E). Interestingly, cleaved caspase-3 immunoreactivity was also present in axons of BTZ-treated SARM1-KO DRG neurons (Figure 6E), even though these axons remained completely intact for more than 96 hours. We also used Western blotting of DRG axonal lysates to examine caspase-3 activation and detected cleaved caspase-3 at 12 and 24 hours after BTZ administration in WT and SARM1-KO axons (Figure 6F). Hence, BTZ-induced axon degeneration is transcriptionally regulated and leads to activation of the effector caspase- 3 in axons, both in WT and SARM1-KO neurons. These findings are consistent with cleaved caspase-3 acting upstream of SARM1 in BTZ-induced axon degeneration. 
A

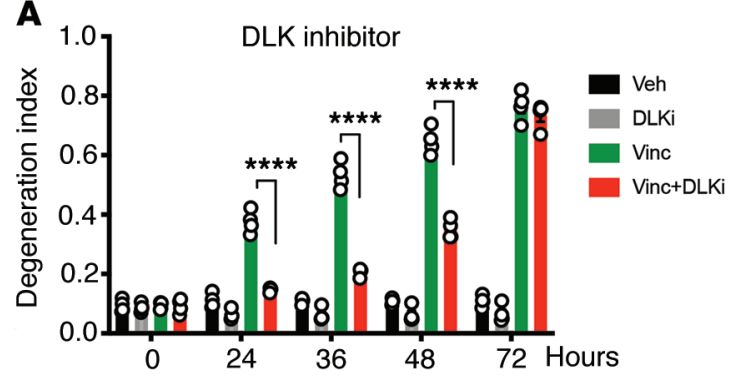

C

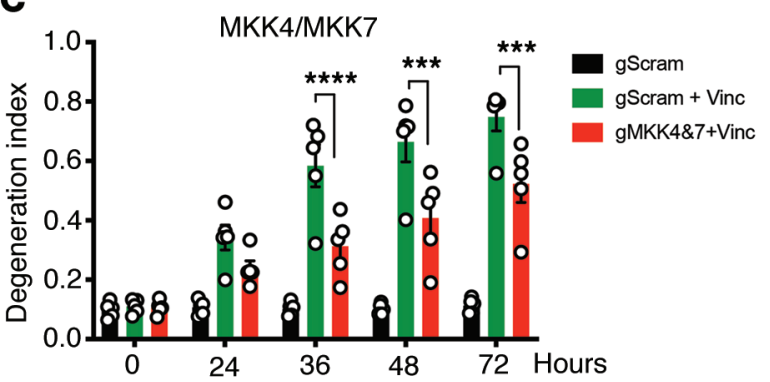

$\mathbf{E}$

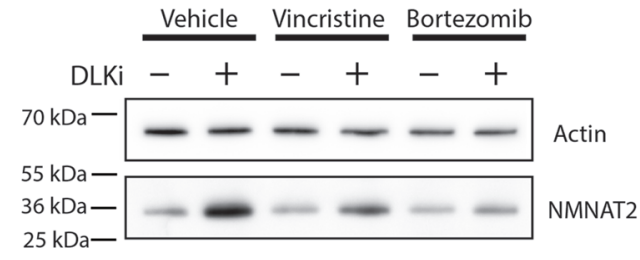

B

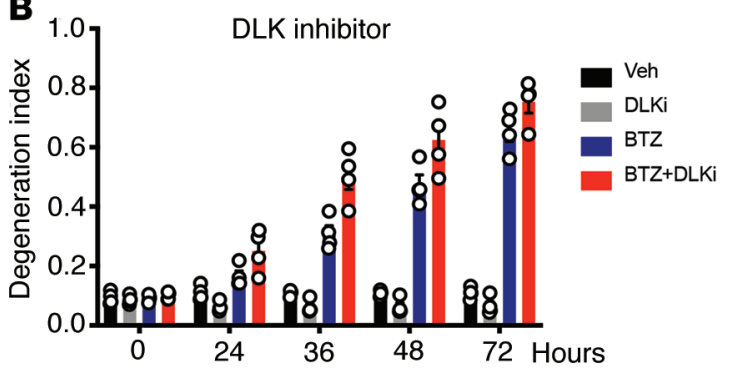

D
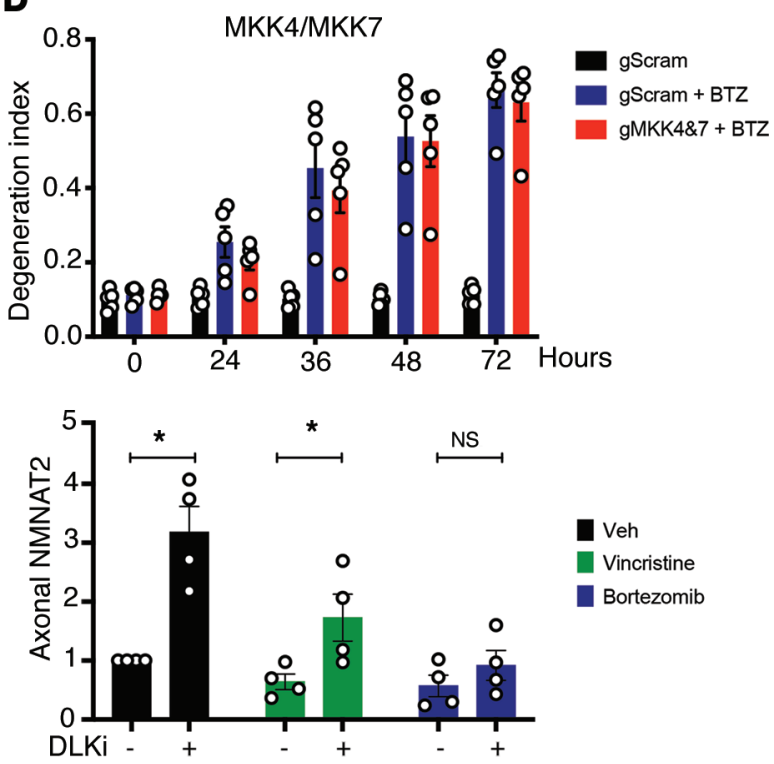

Figure 5. Vincristine- but not BTZ-induced axon degeneration is modulated by a MAPK pathway. (A and B) DRG neurons were treated with $1 \mu M$ of the DLK inhibitor GNE-3511 12 hours before adding vincristine (A) or BTZ (B), and axon degeneration was determined at indicated time points. (A) GNE-3511 decreases vincristine-induced axon degeneration. Two-way ANOVA showed significant effects for group $F(3,12)=352.5, P<0.0001 ;$ time $F(4,48)=515.8, P$ $<0.0001$; and interaction $F(12,48)=205.6, P<0.0001$. Tukey's multiple-comparisons test, ${ }^{* * *} P<0.0001(n=4)$. In contrast, GNE-3511 does not decrease axon degeneration after BTZ administration (B). There was instead a small increase in axon fragmentation $(n=4)$. (C and $\mathbf{D})$ DRG neurons obtained from Cas9-knockin mice expressing guide RNAs to MKK4 and -7 (gMKK4\&7) or scrambled guide RNAs (gScram) were treated with vincristine (C) or BTZ (D), and axon degeneration was determined over time. Inhibiting MKK4 and -7 decreased vincristine-induced axon degeneration (C) but did not affect BTZ-induced axon degeneration (D). A 2-way ANOVA in C showed significant effects of group $F(2,12)=30.64, P<0.0001$; time $F(4,48)=100.2, P<0.0001$; and interaction $F(8,48)=29.5, P<0.0001$. Tukey's multiple-comparisons test, ${ }^{* *} P<0.001 ;{ }^{* * *} P<0.0001(n=5)(\mathbf{C}$ and $\mathbf{D})$. (E) Axon-only extracts derived from DRG neurons treated with the DLK inhibitor GNE-3511 and for 12 hours with vehicle, vincristine, or BTZ were immunoblotted for endogenous NMNAT2. GNE-3511 increased endogenous NMNAT2 12 hours after vehicle and vincristine but not following BTZ administration. Paired $t$ tests, ${ }^{*} P<0.05 ; n=4$.

Loss of SARM1 prevents BTZ-stimulated axon degeneration but not somal damage. Vincristine-induced axon degeneration was axon autonomous (Figure 1, C and E, and ref. 26), whereas that caused by BTZ was mediated by effects on the neuronal cell body. We therefore investigated whether BTZ-induced degeneration is restricted to the axon or also involves the soma. Using Hoechst to stain for live neurons and the ethidium homodimer-1 to mark dead cells, we found that WT DRG cell bodies died over time after BTZ administration (Figure 7A). Interestingly, SARM1-KO cell bodies died over a similar time course as WT DRG neurons after BTZ administration (Figure 7A), although axons remained completely intact for 96 hours and beyond (Figure 7, B and D). Neuronal apoptosis is antagonized by Bcl-XL and overexpression of Bcl-XL prevents neuron death after trophic factor withdrawal and in neurodegenerative models $(41,42)$. To investigate whether expression of an antiapoptotic factor is sufficient to protect from cell body death and axon degeneration after BTZ administration, we used lentivirus to express Bcl-XL in DRG neurons. Although expression of Bcl-XL completely prevented BTZ-induced cell body death (Figure 7, B and C), it did not protect axons as potently as SARM1-KO (Figure 7, B-D). Expressing Bcl-XL in SARM1-KO neurons prevented both cell body death and axon fragmentation (Figure 7, B-D). These data indicate that in cultured DRG neurons, BTZ activates 2 distinct destructive pathways, one that results in death of the soma and the other that results in degeneration of the axon. 

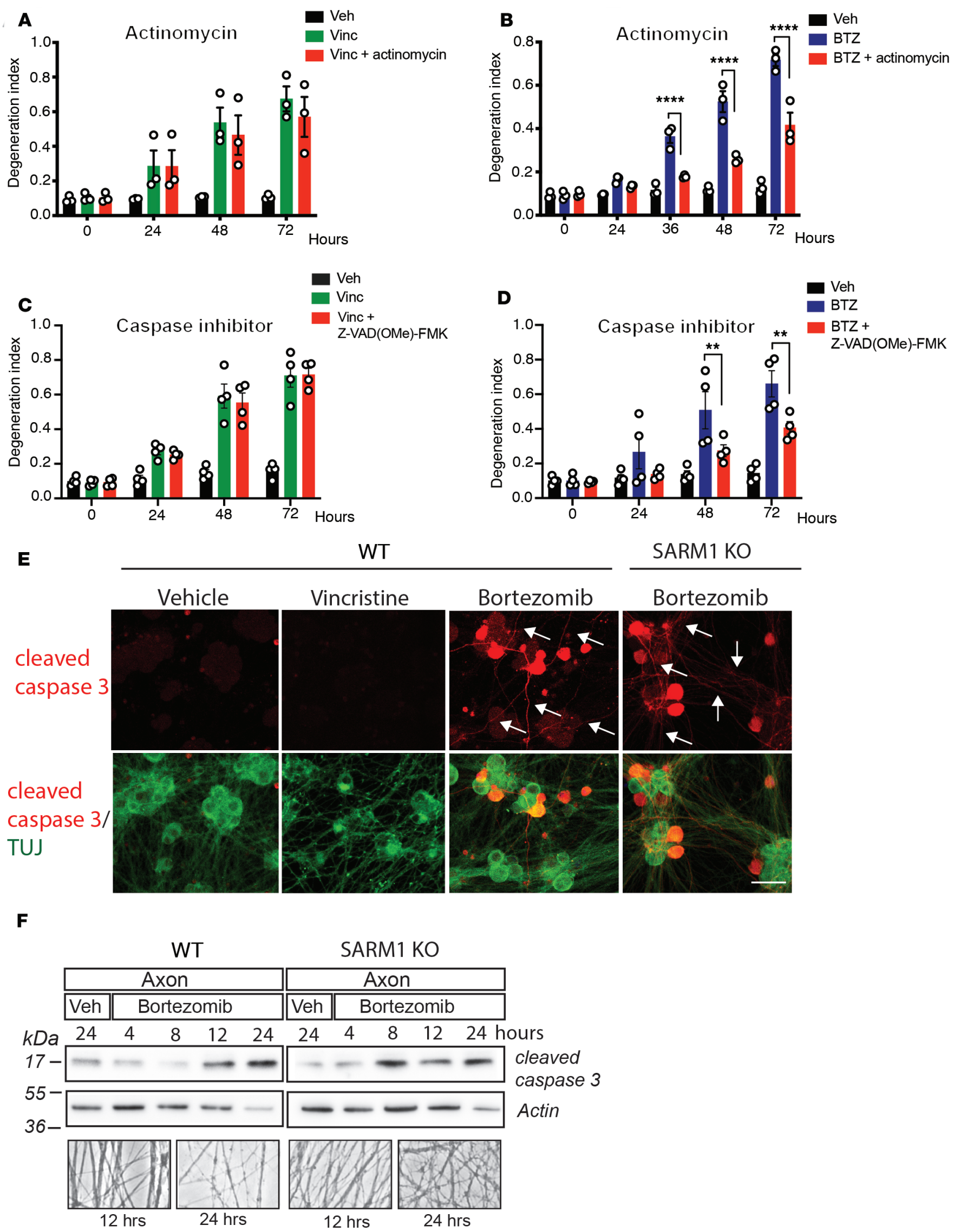

F 
Figure 6. Axon degeneration stimulated by BTZ is transcriptionally regulated and mediated by axonal activated caspases. (A and B) DRG neurons were treated with $1 \mu \mathrm{g} / \mathrm{mL}$ actinomycin starting 1 hour before adding vincristine (A) or BTZ (B), and axon degeneration was determined at indicated time points. Actinomycin decreased axon degeneration after BTZ but not vincristine administration. Two-way ANOVA in B showed significant effects of group $F(2,6)$ $=92.89, P<0.0001$; time $F(4,24)=111.5, P<0.0001$; and interaction $F(8,24)=31.85, P<0.0001$. Tukey's multiple-comparisons test, ${ }^{* * * *} P<0.0001$ ( $n$ $=3$ in $\mathbf{A}$ and B). (C and D) DRG neurons were treated with $100 \mu \mathrm{M}$ of the cell-permeable pan-caspase inhibitor Z-VAD(Ome)-FMK and vincristine (C) or BTZ (D) and axon degeneration was determined. Pan-caspase inhibition decreased BTZ-induced (D) but not vincristine-induced axon degeneration (C). A 2-way ANOVA in D showed significant effects of group $F(2,36)=26.44, P<0.0001$; time $F(3,36)=21.61, P<0.0001$; and interaction $F(6,36)=5.232, P=0.0006$ $n=4$ (C and D). ${ }^{* *} P<0.01$, Tukey's multiple comparison test. (E) Representative microphotographs of DRG neurons stained to label cleaved caspase-3 (red) and counterstained with green fluorescently labeled tubulin III (TUJ) 16 hours after administration of vehicle, vincristine, or BTZ. Cleaved caspase-3 was expressed in axons (white arrows) 16 hours after BTZ but not vincristine administration. Scale bars: $50 \mu \mathrm{m}$. (F) Axon-only lysates derived from WT or SARM1-KO DRG neurons at indicated time points after BTZ or vehicle administration were blotted for endogenous cleaved caspase-3. Actin served as loading control (WT $n=4$; SARM1-KO $n=2$ ). Below are representative phase-contrast photomicrographs of axons just before extraction for immunoblotting demonstrating intact axons 12 and 24 hours after administration of BTZ. Original magnification 200x.

\section{Discussion}

Vincristine and BTZ are among the most effective chemotherapeutic drugs used to treat various cancers, including, but not limited to, lymphomas, leukemias, and multiple myeloma (7). Although vincristine and BTZ have different mechanisms of action, the main nonhematological side effect of both is peripheral neuropathy $(22,23)$, for which no effective treatment exists $(4,5)$. Here, we make the intriguing discovery that the neurodegenerative effects of both vincristine and BTZ converge on a core degeneration program consisting of NMNAT2, SARM1, and $\mathrm{NAD}^{+}$but engage different upstream mechanisms that closely resemble Wallerian degeneration after vincristine and apoptosis after BTZ administration. These 2 axon death pathways are activated in different contexts - Wallerian degeneration leads to axon dismantling after a nerve cut $(8,9)$, whereas apoptosis-induced axon degeneration occurs, for instance, during nervous system development in response to loss of trophic support (43). Although these death pathways share some features, they proceed largely via distinct mechanisms $(37,40)$. For instance, the cell body is required and in fact orchestrates degeneration in apoptosis (38), whereas it is not involved in Wallerian degeneration. Accordingly, the cell body is required for BTZ-induced axon degeneration, whereas degeneration proceeds axon autonomously after vincristine administration (Figure 1 and ref. 26). Furthermore, axon death induced by apoptosis is transcriptionally regulated; is inhibited by antiapoptotic factors, such as Bcl-XL; and leads to caspase activation (43). Similarly, BTZ-induced axon degeneration is transcriptionally regulated, inhibited by overexpression of the antiapoptotic factor Bcl-XL, and mediated by activated axonal caspases. Thus, at least some of the actions of BTZ in DRG neurons are similar to the ones in cancer cells (21) where BTZ induces an apoptosis pathway $(44,45)$. Interestingly, taxol-induced axon loss is also SARM1 dependent (19) and mediated by a cell body-derived signal (46). In contrast, blocking transcription, inhibiting the activation of caspases, or expressing the antiapoptotic factor Bcl-XL do not interfere with Wallerian degeneration or axon degeneration induced by vincristine $(40,47)$. Vincristine interferes with microtubule dynamics, thereby leading to decreased anterograde and retrograde axonal transport (20) similar to, but less severe than, the complete loss of transport that accompanies axotomy. Interventions that prevent axotomy-induced axon degeneration, such as overexpression of NAMPT (28) and inhibition of the MAPK pathway (33-35), also prevent vincristine-induced axon degeneration with similar relative effectiveness. Despite these upstream differences, vincristine and BTZ both lead to a decrease of axonal NMNAT2 levels, activation of SARM1, and depletion of $\mathrm{NAD}^{+}$followed by axon fragmentation. We thus identify NMNAT2/SARM1/NAD ${ }^{+}$as a final common axon destruction pathway that is shared by otherwise distinct axon death programs. Although most work on SARM1 has been done in the context of Wallerian degeneration (9), our finding that SARM1 acts downstream of activated caspases following BTZ is in accord with prior findings that NMNAT and SARM1 act downstream of the apoptotic caspase cascade induced by neurotrophin withdrawal $(12,38,47)$.

Following BTZ administration to cultured DRG neurons, we observed a SARM1-dependent decrease of $\mathrm{NAD}^{+}$in the axon, but not soma, suggesting that SARM1 is activated only in the axon. Accordingly, SARM1-KO prevents BTZ-induced axon degeneration, but not cell death, in vitro. Conversely, overexpressing the antiapoptotic factor Bcl-XL robustly blocks cell body death, but only slows and does not prevent axon degeneration following administration of BTZ, indicating that cell body and axon death are mediated by distinct death pathways and that SARM1 is essential for axon degeneration but not cell body death following BTZ. The same dichotomy is observed in apoptosis-induced degeneration following NGF withdrawal in which expression of cytNMNAT1 (which inhibits SARM1 function) protects from NGF-induced axon degeneration but does not block cell death (47). 
A

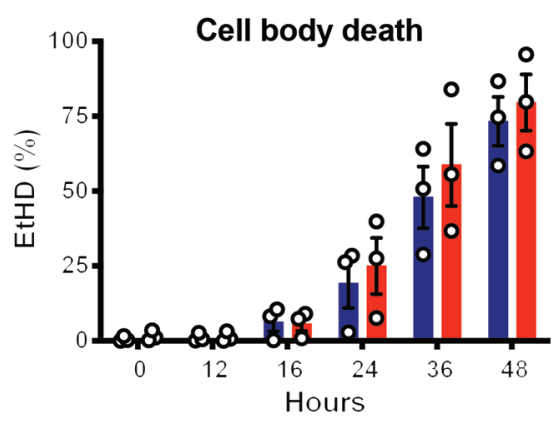

WT

SARM1KO

B

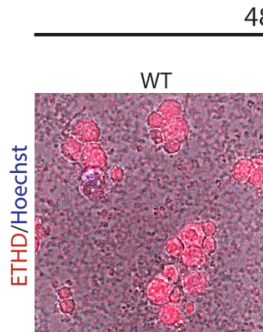

48 hrs BTZ

96 hrs BTZ
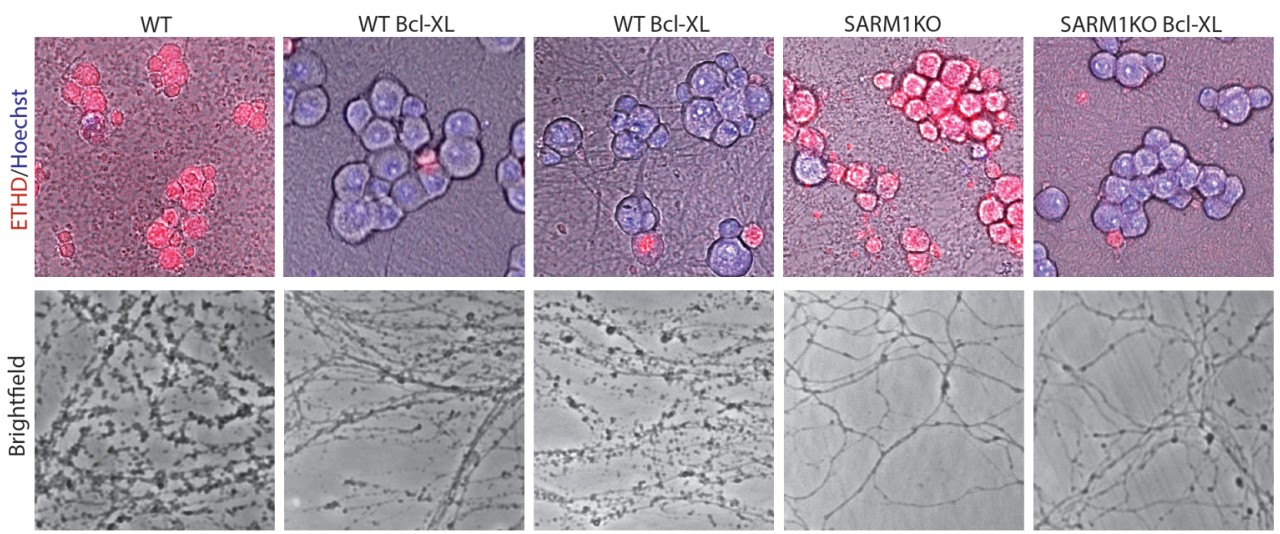

C 96 hours Bortezomib

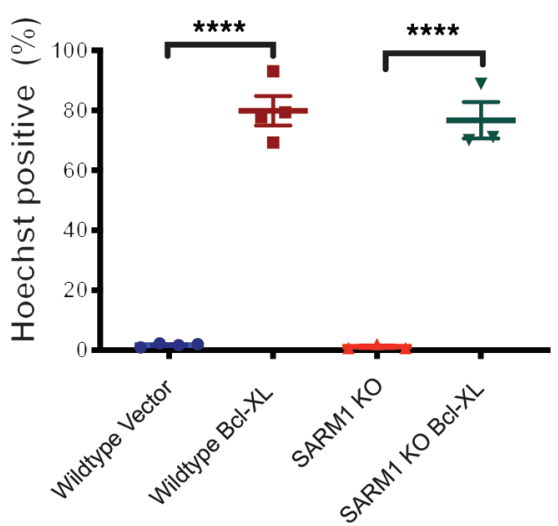

D

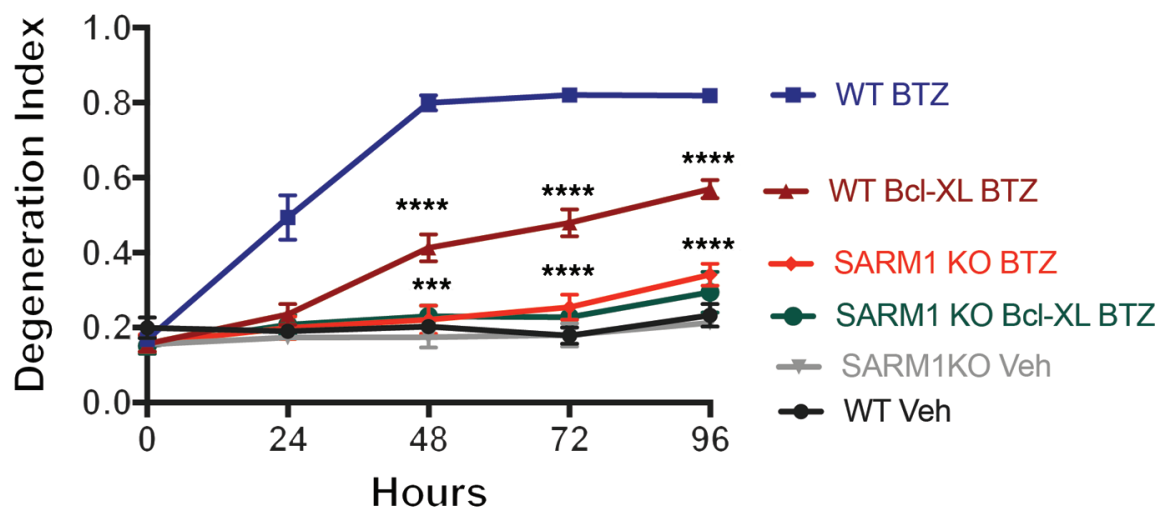

Figure 7. Loss of SARM1 prevents BTZ-stimulated axon degeneration but not cell death. (A) WT and SARM1-KO DRG neurons were treated with BTZ and incubated with Hoechst and ethidium homodimer (ETHD positive) at indicated time points to stain, respectively, live and dead cells. After BTZ administration, the number of dead (ETHD) WT and SARM1-KO DRG neurons increased over time to the same extent. A 2-way ANOVA showed a significant effect of time $F(5,20)=72.27, P<0.0001$, but not group $F(1,4)=0.2639, P=0.6345$, or interaction $F(5,20)=0.3648, P=0.8666 ; n=3$. (B) Representative photomicrographs of WT and SARM1-KO DRG neurons expressing Bcl-XL or vector and treated with BTZ for 48 or 96 hours that were incubated with ETHD (red) and Hoechst (blue) to label dead (ETHD) and living (Hoechst) cells. Expression of Bcl-XL prevented neuronal death in both WT and SARM1-KO neurons. Phase-contrast photomicrographs of axons corresponding to the cells above, showing that expression of Bcl-XL did not prevent axon degeneration in WT DRG neurons as potently as SARM1 KO. (C) WT and SARM1-KO DRG neurons expressing control vector or Bcl-XL were stained with ETHD and Hoechst 96 hours after BTZ was added. One-way ANOVA $F(3,10)=128.6, P<0.0001 ; *^{* * *} P<0.0001$. $n=4$ WT; $n=3$ SARM1KO. (D) Axon degeneration of experiments shown in C. WT and SARM1-KO DRG expressing indicated constructs were treated with BTZ or vehicle, and axon degeneration was determined at indicated time points. Two-way ANOVA showed significant effects of group $F(5,69)=165.5, P<0.0001$; time $F$ $(4,69)=67, P<0.0001$; and interaction $F(20,69)=14.99, P<0.0001$; Tukey's multiple-comparisons test ${ }^{* * *} P<0.001$ WT BTZ Bcl-XL vs. SARM1-KO $\mathrm{BCl}-\mathrm{XL} ;{ }^{* * *} \mathrm{P}<0.0001 ; n=4 \mathrm{WT} ; n=3$ SARM1-KO. 
Furthermore, SARM1-KO prevents axon degeneration in vivo after optic nerve crush but does not mitigate the accompanying retinal ganglion cell death (48).

Whether DRG neurons degenerate in patients following BTZ administration is unknown. Large multicenter phase II and III studies have shown that BTZ-induced neuropathy resolves or improves in many patients after treatment has ended (23), suggesting that cell body death is not a major contributing factor in BTZ-induced neuropathy. This conclusion is consistent with our in vivo SARM1-KO result showing substantial protection from BTZ-induced axon degeneration. Nevertheless, degeneration of DRG neurons in a patient subset that continues to experience neuropathic symptoms after discontinuation of BTZ cannot be ruled out (49). Indeed, in a study of BTZ-treated mice, Carozzi et al. observed cytoplasmic condensation and degeneration of DRG neurons (50). Thus, it is possible that for some patients a combination therapy targeting the cell body and axon may lead to best results.

A major finding of our study is that targeting the core axon degeneration program either by maintaining $\mathrm{NAD}^{+}$or inhibiting SARM1 completely and persistently blocks axon degeneration after BTZ and vincristine in vitro and in vivo. In addition, SARM1-KO or NR supplementation prevent paclitaxel-induced neuropathy and diabetic neuropathy in mouse models $(19,51,52)$, indicating that targeting the core axon degeneration program attenuates a variety of forms of peripheral neuropathy. $\mathrm{NAD}^{+}$can be increased in human patients by the naturally occurring supplement vitamin $\mathrm{B}_{3}(53,54)$. Although increasing $\mathrm{NAD}^{+}$may be helpful for some forms of neuropathy, it could be problematic in the setting of certain cancers, such as, for instance, multiple myeloma cells, in which intracellular $\mathrm{NAD}^{+}$levels can influence treatment efficacy (55). Because SARM1 is predominantly expressed in neurons and kept inhibited at rest, therapeutically inhibiting it is less likely to be associated with such negative effects on cancer therapy. We recently discovered that SARM1 has enzymatic activity (16), which provides a novel target for the rational design of inhibitors and developed a SARM1-DN transgene (27) that potently prevents vincristine- (27) and BTZ-induced axon degeneration in vitro. We suggest that inhibiting SARM1 may be of therapeutic value to prevent different chemotherapy-induced neuropathies and possibly other peripheral polyneuropathies.

\section{Methods}

This manuscript was prepared in adherence to the Animal Research: Reporting of In Vivo Experiments guidelines. Pregnant CD1 mice were purchased from Charles River. C57BL/6 mice were purchased from The Jackson Laboratory. SARM1-KO mice were a gift from Marco Colonna (56) and bred in our colony. Male and female mice were used for all in vitro experiments. For in vivo studies, male mice aged 10-16 weeks were used. Chemicals were purchased from MilliporeSigma unless indicated otherwise.

Mouse embryonic DRG cultures. Embryonic dorsal root ganglia were obtained from embryonic day 13.5 CD1 (Charles River) or SARM1-KO mice. Cells were dissociated by incubation in $0.05 \%$ Trypsin-EDTA (Gibco) at $37^{\circ} \mathrm{C}$ and resuspended in Neurobasal medium (Invitrogen) containing 2\% B27 (Invitrogen), $50 \mathrm{ng} / \mathrm{mL}$ NGF (Harlan Laboratories), $1 \mu \mathrm{M}$ 5-Fluoro-2'-deoxyuridine (MilliporeSigma), and $1 \mu \mathrm{M}$ uridine and plated in 48-well plates coated with poly-D-lysine and laminin. Cultures were maintained in Neurobasal medium (Invitrogen) supplemented with 2\% B27 (Invitrogen), $50 \mathrm{ng} / \mathrm{mL}$ NGF (Harlan Laboratories), and $1 \mathrm{mM}$ 5-Fluoro-2'-deoxyuridine (MilliporeSigma).

Microfluidic cultures. Experiments described in Figure 1, C-E, were performed in microfluidic chambers. Microfluidic chambers, specifically AXIS AX50010TC Axon Isolation Device (MilliporeSigma), were placed onto poly-D-lysine- and laminin-coated 6-well plates. Cell suspension was pipetted into each side of the upper channel of the microfluidic device. Medium was exchanged with fresh every other day. On the day of the experiment (DIV 5-7), a hydrostatic gradient was introduced, and drugs were added to the compartment with the lower hydrostatic pressure. Bright-field images of cells growing in microfluidic chambers were obtained using an Olympus CKX41 microscope with a Nikon DS-Qi1 camera.

Drugs and reagents. All drugs and reagents were purchased from MilliporeSigma unless noted. On DIV 7, vincristine sulfate (40 $\mathrm{nM}$ in DMSO), BTZ (100 nM in DMSO), or vehicle (DMSO) was added to cultured DRG neurons and remained in the wells until the end of the experiments. NR (1 mM), a gift from ChromaDex Inc., and DLK inhibitor (GNE-3511, Calbiochem; $1 \mu \mathrm{M}$ in DMSO) were added 12 hours before the start of the experiment. The caspase inhibitor Z-VAD(OMe)-FMK (Enzo Life Sciences; $100 \mu \mathrm{M})$ was added 1 hour before the start of the experiment. Actinomycin-D $(1 \mu \mathrm{g} / \mathrm{mL})$ was added at the start of the experiment. TMRM (Thermo Fisher Scientific) was added to the wells and incubated for 30 minutes. 
The TMRM and bright-field images were imaged simultaneously using an Olympus CKX41 microscope with a Nikon DS-Qil camera.

Lentiviral preparation and transduction. Mammalian expression constructs were derived from an FCIV lentiviral vector (32) containing a ubiquitin promoter and Venus marker. HEK293T cells were maintained in DMEM (Gibco) supplemented with 10\% FBS (Peak Serum) and 2 mM L-glutamine (DFG). At approximately $80 \%$ confluence, cells were transfected with lentiviral packaging plasmid psPAX2, vesicular stomatitis virus glycoprotein, and lentiviral expression vector FCIV. Lentiviral particles were collected 48 hours later and concentrated with Lenti-X concentrator (Clontech) to a final concentration of $1 \times 10^{6}$ infectious particles/mL. Embryonic DRG cultures were infected with FCIV control, cytNMNAT1 (32), NRK1 (28), NAMPT (28), or a SARM1-DN mutant (SARM1-K193R/H685A/H194A; ref. 27). Lentiviral particles were added directly to neuronal media $\left(10^{5}\right.$ particles $\left./ \mathrm{mL}\right)$ the day after plating (DIV 1$)$. Lentiviral expression of constructs was confirmed by positive fluorescent signal in the cell bodies.

Axon degeneration imaging and index measurement. DRG neurons were grown in 48-well spot cultures. Before the start of each experiment, and at time points throughout, images were obtained with an Operetta High-Content Imaging System (PerkinElmer) using a $\times 20$ long working range objective. A total of $15-20$ images per well were collected, from a minimum of 3 wells per treatment condition. These images were then analyzed in ImageJ software (NIH) using a custom-made "degeneration index," which gives a numerical output corresponding to the fractional area of fragmented axons (24). Higher degeneration index values indicate more degenerated axons.

Immunohistochemistry. DRG cultures (on DIV 7) were fixed in 4\% paraformaldehyde (Electron Microscopy Sciences) for 20 minutes followed by PBS rinse and immunostaining. Briefly, cultures were blocked in blocking solution (5\% normal goat serum and 0.1\% Triton X-100 in PBS) for 1 hour and then incubated overnight in primary antibody at $4{ }^{\circ} \mathrm{C}$. The following primary antibodies were used: Alexa Fluor 488 antitubulin $\beta_{3}$ (1:1000; BioLegend; 801203) and rabbit anti-cleaved caspase-3 (1:500; Cell Signaling Technology; 9661). Samples were washed 3 times with $0.1 \%$ Triton X-100 in PBS and incubated with Alexa Fluor 594-conjugated goat antirabbit (1:500; Invitrogen; A-11032) for 2 hours at room temperature. Images were captured with a Leica DFC 7000-T Camera on a Leica DMI 4000B microscope using a $\times 20$ objective.

Western blotting. Cell and axon lysates were collected in sample buffer (1 M Tris-HCL, glycerol, SDS, bromophenol blue), loaded into Mini-PROTEAN precast gels (Bio-Rad), and resolved via SDS-PAGE using a Mini-PROTEAN Tetra Vertical Electrophoresis Cell (Bio-Rad). The gel was then transferred onto a 0.2- $\mu \mathrm{M}$ nitrocellulose membrane using the Trans-Blot Turbo Transfer System (Bio-Rad). The membrane was washed, blocked, and incubated overnight with primary antibody. The following primary antibodies were used: rabbit anti-cleaved caspase-3 (1:500; Cell Signaling Technology; 9661), rabbit anti-caspase-3 (1:1000; Cell Signaling Technology; 9662), rabbit anti- $\beta$-actin (1:1000; Cell Signaling Technology; 4970), and mouse anti-NMNAT2 (1:250; Santa Cruz Biotechnology; sc-515206). The following day, HRP-conjugated antimouse or antirabbit secondary antibodies (1:10,000; Jackson ImmunoResearch; 115-035-003 and 111-035-144) were incubated for 1 hour and were detected by WesternBright Sirius HRP Substrate (Advansta). Western blot quantification was performed using ImageJ (NIH).

$N A D^{+}$measurement. Axons and cell bodies were lysed by addition of $0.5 \mathrm{M}$ perchloric acid. Extracts were centrifuged and supernatants were collected, neutralized with $3 \mathrm{M} \mathrm{K}_{2} \mathrm{CO}_{3}$, and diluted in potassium phosphate buffer. NAD ${ }^{+}$was assayed by HPLC on an LC-18T HPLC column (Supelco) at a flow rate of $1 \mathrm{~mL} / \mathrm{min}$. Elution peaks were matched to $\mathrm{NAD}^{+}$standards. For experiments involving NRK, neurons were transduced with concentrated virus expressing NRK on DIV 1, and NR was added 12 hours before adding vincristine or BTZ.

BTZ-induced peripheral neuropathy. BTZ (LC Laboratories B-1408) was dissolved in 100\% ethanol/ Tween- $80 /$ normal saline $(5 \% / 5 \% / 90 \% v / v)$ and administered by tail vein injections at a dose of $0.8 \mathrm{mg} / \mathrm{kg}$ twice a week for 4 weeks to SARM1-KO mice $(n=7)$ and WT C57BL/6J mice $(n=7)$. Saline was administered at the same volume, route, and frequency to SARM1-KO $(n=5)$ and C57BL/6J $(n=5)$ mice. Four days after the last dose, mice were euthanized by isoflurane inhalation, and footpads were harvested for the evaluation of IENFs. Two-millimeter punch biopsies of medial plantar footpads were fixed with paraformaldehyde-lysine-periodate for 24 hours at $4^{\circ} \mathrm{C}$ and transferred into a cryoprotective solution, and $50-\mu \mathrm{m}$ thick serial sections were cut with a Thermo Scientific Microm HM550 freezing microtome. The sections were stained with the pan-axonal marker anti-protein gene product 9.5 (Bio-Rad, 7863-0504). IENFs were counted in 3 sections for each animal by observers blind to treatment according to standard protocols as described (57) and averaged using a microscope at $\times 40$ magnification for each animal. 
Statistics. Unless otherwise stated, data are reported as mean \pm SEM. Between group comparisons were made with 1-way or 2-way ANOVA as appropriate. Two-sided significance tests were used throughout, and $P<0.05$ was considered statistically significant. All statistics were calculated with the aid of GraphPad Prism software.

Study approval. All experiments were performed in accordance with guidelines mandated by the NIH Guide for the Care and Use of Laboratory Animals (National Academies Press, 2011) and were approved by the Washington University in St. Louis Medical School Institutional Animal Care and Use Committee (protocols 20170030 and 20180030) and the Johns Hopkins Institutional Animal Care and Use Committees (protocol M015M322).

\section{Author contributions}

$\mathrm{SG}, \mathrm{AH}$, and $\mathrm{AD}$ designed the research studies. SG, RAD, GCC, and SXH conducted in vitro experiments and analyzed the data. ACF and AH conducted in vivo experiments and analyzed the data. SG, AH, JM, and $\mathrm{AD}$ obtained funding for the study. SG, JM, and $\mathrm{AD}$ wrote the manuscript, and all authors edited and revised the manuscript.

\section{Acknowledgments}

We thank members of the Milbrandt and DiAntonio labs (Washington University) for sharing of reagents and fruitful discussions. This work was supported by NIH grants K08NS091448 (to SG); R01CA219866, R01NS087632, and R01CA218263 (to JM and AD); and R01NS091260 (to AH) as well as the Thompson Family Foundation Initiative grant PDS 155352 (to SG, AD, and JM) and the Dr. Miriam and Sheldon G. Adelson Medical Foundation (to AH).

Address correspondence to: Aaron DiAntonio, Department of Developmental Biology, Washington University Medical School in St. Louis, 4523 Clayton Avenue, CB 8103, St. Louis, Missouri 63110, USA. Phone: 314.362.9925; diantonio@wustl.edu. Or to: Jeffrey Milbrandt, Department of Genetics, Washington University Medical School in St. Louis, 660 South Euclid Avenue, Box 8232, St. Louis, Missouri 63110, USA. Phone: 314.362.2139; Email: jmilbrandt@wustl.edu. Or to: Stefanie Geisler, Department of Neurology, Washington University Medical School in St. Louis, 660 South Euclid Avenue, Box 8111, St. Louis, Missouri 63110, USA. Phone: 314.362.4955; Email: geislers@wustl.edu.

RAD's present address is: The Vollum Institute, Oregon Health \& Science University, Portland, Oregon, USA.

1. Argyriou AA, Kyritsis AP, Makatsoris T, Kalofonos HP. Chemotherapy-induced peripheral neuropathy in adults: a comprehensive update of the literature. Cancer Manag Res. 2014;6:135-147.

2. Shah A, et al. Incidence and disease burden of chemotherapy-induced peripheral neuropathy in a population-based cohort. J Neurol Neurosurg Psychiatry. 2018;89(6):636-641.

3. Boyette-Davis JA, et al. Persistent chemoneuropathy in patients receiving the plant alkaloids paclitaxel and vincristine. Cancer Chemother Pharmacol. 2013;71(3):619-626.

4. Hershman DL, et al. Prevention and management of chemotherapy-induced peripheral neuropathy in survivors of adult cancers: American Society of Clinical Oncology clinical practice guideline. J Clin Oncol. 2014;32(18):1941-1967.

5. Majithia N, Temkin SM, Ruddy KJ, Beutler AS, Hershman DL, Loprinzi CL. National Cancer Institute-supported chemotherapy-induced peripheral neuropathy trials: outcomes and lessons. Support Care Cancer. 2016;24(3):1439-1447.

6. Fukuda Y, Li Y, Segal RA. A mechanistic understanding of axon degeneration in chemotherapy-induced peripheral neuropathy. Front Neurosci. 2017;11:481.

7. Staff NP, Grisold A, Grisold W, Windebank AJ. Chemotherapy-induced peripheral neuropathy: a current review. Ann Neurol. 2017;81(6):772-781

8. Conforti L, Gilley J, Coleman MP. Wallerian degeneration: an emerging axon death pathway linking injury and disease. Nat Rev Neurosci. 2014;15(6):394-409.

9. Gerdts J, Summers DW, Milbrandt J, DiAntonio A. Axon self-destruction: new links among SARM1, MAPKs, and NAD ${ }^{+}$ metabolism. Neuron. 2016;89(3):449-460.

10. DiAntonio A. Axon degeneration: mechanistic insights lead to therapeutic opportunities for the prevention and treatment of peripheral neuropathy. Pain. 2019;160(suppl 1):S17-S22.

11. Osterloh JM, et al. dSarm/Sarm1 is required for activation of an injury-induced axon death pathway. Science. 2012;337(6093):481-484.

12. Gerdts J, Summers DW, Sasaki Y, DiAntonio A, Milbrandt J. Sarm1-mediated axon degeneration requires both SAM and TIR interactions. J Neurosci. 2013;33(33):13569-13580.

13. Di Stefano $\mathrm{M}$, et al. A rise in NAD precursor nicotinamide mononucleotide $(\mathrm{NMN})$ after injury promotes axon degeneration. 
Cell Death Differ. 2015;22(5):731-742.

14. Zhao ZY, et al. A cell-permeant mimetic of NMN activates SARM1 to produce cyclic ADP-ribose and induce non-apoptotic cell death. iScience. 2019;15:452-466.

15. Liu HW, et al. Pharmacological bypass of $\mathrm{NAD}^{+}$salvage pathway protects neurons from chemotherapy-induced degeneration. Proc Natl Acad Sci U S A. 2018;115(42):10654-10659.

16. Essuman K, Summers DW, Sasaki Y, Mao X, DiAntonio A, Milbrandt J. The SARM1 Toll/interleukin-1 receptor domain possesses intrinsic $\mathrm{NAD}^{+}$cleavage activity that promotes pathological axonal degeneration. Neuron. 2017;93(6):1334-1343.e5.

17. Gerdts J, Brace EJ, Sasaki Y, DiAntonio A, Milbrandt J. SARM1 activation triggers axon degeneration locally via NAD ${ }^{+}$ destruction. Science. 2015;348(6233):453-457.

18. Geisler S, Doan RA, Strickland A, Huang X, Milbrandt J, DiAntonio A. Prevention of vincristine-induced peripheral neuropathy by genetic deletion of SARM1 in mice. Brain. 2016;139(pt 12):3092-3108.

19. Turkiew E, Falconer D, Reed N, Höke A. Deletion of Sarm1 gene is neuroprotective in two models of peripheral neuropathy. J Peripher Nerv Syst. 2017;22(3):162-171.

20. LaPointe NE, Morfini G, Brady ST, Feinstein SC, Wilson L, Jordan MA. Effects of eribulin, vincristine, paclitaxel and ixabepilone on fast axonal transport and kinesin-1 driven microtubule gliding: implications for chemotherapy-induced peripheral neuropathy. Neurotoxicology. 2013;37:231-239.

21. Meregalli C, et al. Evaluation of tubulin polymerization and chronic inhibition of proteasome as citotoxicity mechanisms in bortezomib-induced peripheral neuropathy. Cell Cycle. 2014;13(4):612-621.

22. DeAngelis LM, Gnecco C, Taylor L, Warrell RP. Evolution of neuropathy and myopathy during intensive vincristine/corticosteroid chemotherapy for non-Hodgkin's lymphoma. Cancer. 1991;67(9):2241-2246.

23. Richardson PG, et al. Single-agent bortezomib in previously untreated multiple myeloma: efficacy, characterization of peripheral neuropathy, and molecular correlations with response and neuropathy. J Clin Oncol. 2009;27(21):3518-3525.

24. Sasaki Y, Vohra BP, Lund FE, Milbrandt J. Nicotinamide mononucleotide adenylyl transferase-mediated axonal protection requires enzymatic activity but not increased levels of neuronal nicotinamide adenine dinucleotide. $J$ Neurosci. 2009;29(17):5525-5535.

25. Vaur $\mathrm{P}$, et al. Nicotinamide riboside, a form of vitamin $\mathrm{B}_{3}$, protects against excitotoxicity-induced axonal degeneration. FASEB J. 2017;31(12):5440-5452.

26. Silva A, Wang Q, Wang M, Ravula SK, Glass JD. Evidence for direct axonal toxicity in vincristine neuropathy. J Peripher Nerv Syst. 2006;11(3):211-216.

27. Geisler S, et al. Gene therapy targeting SARM1 blocks pathological axon degeneration in mice. J Exp Med. 2019;216(2):294-303.

28. Sasaki Y, Nakagawa T, Mao X, DiAntonio A, Milbrandt J. NMNAT1 inhibits axon degeneration via blockade of SARM1-mediated NAD ${ }^{+}$depletion. Elife. 2016;5:e19749.

29. Bieganowski P, Brenner C. Discoveries of nicotinamide riboside as a nutrient and conserved NRK genes establish a Preiss-Handler independent route to NAD+ in fungi and humans. Cell. 2004;117(4):495-502.

30. Gilley J, Orsomando G, Nascimento-Ferreira I, Coleman MP. Absence of SARM1 rescues development and survival of NMNAT2-deficient axons. Cell Rep. 2015;10(12):1974-1981.

31. Gilley J, Coleman MP. Endogenous Nmnat2 is an essential survival factor for maintenance of healthy axons. PLoS Biol. 2010;8(1):e1000300.

32. Sasaki Y, Araki T, Milbrandt J. Stimulation of nicotinamide adenine dinucleotide biosynthetic pathways delays axonal degeneration after axotomy. J Neurosci. 2006;26(33):8484-8491.

33. Miller BR, Press C, Daniels RW, Sasaki Y, Milbrandt J, DiAntonio A. A dual leucine kinase-dependent axon self-destruction program promotes Wallerian degeneration. Nat Neurosci. 2009;12(4):387-389.

34. Yang J, et al. Pathological axonal death through a MAPK cascade that triggers a local energy deficit. Cell. 2015;160(1-2):161-176

35. Walker LJ, Summers DW, Sasaki Y, Brace EJ, Milbrandt J, DiAntonio A. MAPK signaling promotes axonal degeneration by speeding the turnover of the axonal maintenance factor NMNAT2. Elife. 2017;6:e22540.

36. Summers DW, Milbrandt J, DiAntonio A. Palmitoylation enables MAPK-dependent proteostasis of axon survival factors. Proc Natl Acad Sci U S A. 2018;115(37):E8746-E8754.

37. Geden MJ, Deshmukh M. Axon degeneration: context defines distinct pathways. Curr Opin Neurobiol. 2016;39:108-115.

38. Simon DJ, et al. Axon degeneration gated by retrograde activation of somatic pro-apoptotic signaling. Cell. 2016;164(5):1031-1045

39. Simon DJ, et al. A caspase cascade regulating developmental axon degeneration. J Neurosci. 2012;32(49):17540-17553.

40. Finn JT, Weil M, Archer F, Siman R, Srinivasan A, Raff MC. Evidence that Wallerian degeneration and localized axon degeneration induced by local neurotrophin deprivation do not involve caspases. J Neurosci. 2000;20(4):1333-1341.

41. Garcera A, et al. A new model to study spinal muscular atrophy: neurite degeneration and cell death is counteracted by BCL$\mathrm{X}(\mathrm{L})$ overexpression in motoneurons. Neurobiol Dis. 2011;42(3):415-426.

42. Mincheva-Tasheva S, Obis E, Tamarit J, Ros J. Apoptotic cell death and altered calcium homeostasis caused by frataxin depletion in dorsal root ganglia neurons can be prevented by BH4 domain of Bcl-xL protein. Hum Mol Genet. 2014;23(7):1829-1841.

43. Pease SE, Segal RA. Preserve and protect: maintaining axons within functional circuits. Trends Neurosci. 2014;37(10):572-582.

44. Berges C, et al. Proteasome inhibition activates the mitochondrial pathway of apoptosis in human CD4 ${ }^{+} \mathrm{T}$ cells. J Cell Biochem. 2009;108(4):935-946.

45. Ding WX, Ni HM, Chen X, Yu J, Zhang L, Yin XM. A coordinated action of Bax, PUMA, and p53 promotes MG132-induced mitochondria activation and apoptosis in colon cancer cells. Mol Cancer Ther. 2007;6(3):1062-1069.

46. Pease-Raissi SE, et al. Paclitaxel reduces axonal Bclw to initiate $\mathrm{IP}_{3}$ R1-dependent axon degeneration. Neuron. 2017;96(2):373-386.e6.

47. Vohra BP, Sasaki Y, Miller BR, Chang J, DiAntonio A, Milbrandt J. Amyloid precursor protein cleavage-dependent and -independent axonal degeneration programs share a common nicotinamide mononucleotide adenylyltransferase 1-sensitive pathway. J Neurosci. 2010;30(41):13729-13738.

48. Fernandes KA, et al. Role of SARM1 and DR6 in retinal ganglion cell axonal and somal degeneration following axonal injury. Exp Eye Res. 2018;171:54-61.

49. Cata JP, Weng HR, Burton AW, Villareal H, Giralt S, Dougherty PM. Quantitative sensory findings in patients with bortezo- 
mib-induced pain. J Pain. 2007;8(4):296-306.

50. Carozzi VA, et al. Neurophysiological and neuropathological characterization of new murine models of chemotherapy-induced chronic peripheral neuropathies. Exp Neurol. 2010;226(2):301-309.

51. Hamity MV, White SR, Walder RY, Schmidt MS, Brenner C, Hammond DL. Nicotinamide riboside, a form of vitamin B3 and $\mathrm{NAD}^{+}$precursor, relieves the nociceptive and aversive dimensions of paclitaxel-induced peripheral neuropathy in female rats. Pain. 2017;158(5):962-972.

52. Trammell SA, et al. Nicotinamide riboside opposes type 2 diabetes and neuropathy in mice. Sci Rep. 2016;6:26933.

53. Martens CR, et al. Chronic nicotinamide riboside supplementation is well-tolerated and elevates NAD ${ }^{+}$in healthy middle-aged and older adults. Nat Commun. 2018;9(1):1286.

54. Trammell SA, et al. Nicotinamide riboside is uniquely and orally bioavailable in mice and humans. Nat Commun. 2016;7:12948.

55. Cagnetta A, et al. Intracellular NAD ${ }^{+}$depletion enhances bortezomib-induced anti-myeloma activity. Blood. 2013;122(7):1243-1255.

56. Szretter KJ, Samuel MA, Gilfillan S, Fuchs A, Colonna M, Diamond MS. The immune adaptor molecule SARM modulates tumor necrosis factor alpha production and microglia activation in the brainstem and restricts West Nile Virus pathogenesis. J Virol. 2009;83(18):9329-9338.

57. Melli G, Jack C, Lambrinos GL, Ringkamp M, Höke A. Erythropoietin protects sensory axons against paclitaxel-induced distal degeneration. Neurobiol Dis. 2006;24(3):525-530. 